\title{
Design and Synthesis of Novel Pyridinium Chiral Ionic Liquids Tethered to a Urea Functionality
}

\author{
Bukuo Ni, Qianying Zhang and Allan D. Headley* \\ Department of Chemistry, Texas A\&M University-Commerce \\ Commerce, TX 75429-3011, USA \\ E-mail; allan_headley@TAMU-Commerce.edu
}

\section{Supporting Information}

Table of Contents

\begin{tabular}{c|c}
\hline Analytical data for compounds (S)-5-(S)-6 & S2 \\
\hline Analytical data for compounds (S)-8-(S)-12c & S2-S5 \\
\hline${ }^{1}$ H NMR and ${ }^{13}$ C NMR spectra of those compounds & S6-S35 \\
\hline
\end{tabular}


(1) Compound (S)-5: Yield: $86 \%$. White solid; mp: $79-80^{\circ} \mathrm{C}$; $[\alpha]_{\mathrm{D}}^{20}=-8.56^{\circ}(\mathrm{c}=1.39$, EtOH); ${ }^{1} \mathrm{H}$ NMR $\left(400 \mathrm{MHz}, \mathrm{CDCl}_{3}\right) \delta 8.49(\mathrm{~d}, J=4.4 \mathrm{~Hz}, 1 \mathrm{H}), 7.64(\mathrm{td}, J=8.0$ and 1.6 $\mathrm{Hz}, 1 \mathrm{H}), 7.33-7.23(\mathrm{~m}, 1 \mathrm{H}), 7.16(\mathrm{dd}, J=6.8$ and $5.2 \mathrm{~Hz}, 1 \mathrm{H}), 5.96-5.84(\mathrm{~m}, 1 \mathrm{H}), 5.48$ (d, $J=8 \mathrm{~Hz}, 1 \mathrm{H}), 4.54-4.42(\mathrm{~m}, 3 \mathrm{H}), 3.70(\mathrm{~s}, 3 \mathrm{H}), 1.76-1.43(\mathrm{~m}, 3 \mathrm{H}), 0.92(\mathrm{dd}, J=6.4$ and $5.2 \mathrm{~Hz}, 6 \mathrm{H}) .{ }^{13} \mathrm{C} \mathrm{NMR}\left(100 \mathrm{MHz}, \mathrm{CDCl}_{3}\right) \delta 175.1,158.1,158.0,148.8,136.8,122.2$, $122.1,52.1,51.6,45.5,41.8,24.8,22.8,21.9 ; \quad I R$ (neat) $v=3748,1736,1650,1634$, 1558, $1195 \mathrm{~cm}^{-1}$; Anal. Calcd for $\mathrm{C}_{14} \mathrm{H}_{21} \mathrm{~N}_{3} \mathrm{O}_{3}: \mathrm{C}, 60.20 \%$; $\mathrm{H}, 7.58 \%$; N, $15.04 \%$, found C, $60.09 \%, \mathrm{H}, 7.78 \%, \mathrm{~N}, 14.99 \%$.

(2) Compound (S)-6: Yield: $90 \%$. White solid; mp: $98-99{ }^{\circ} \mathrm{C} ;[\alpha]_{\mathrm{D}}{ }^{20}=20^{\circ}(\mathrm{c}=0.99$, EtOH); ${ }^{1} \mathrm{H}$ NMR (400 MHz, $\left.\mathrm{CDCl}_{3}\right) \delta 8.41(\mathrm{~d}, J=4.8 \mathrm{~Hz}, 1 \mathrm{H}), 7.62(\mathrm{t}, J=8.0 \mathrm{~Hz}, 1 \mathrm{H})$, 7.39-6.96 (m, 7H), 6.04-5.72 (br, 1H), $5.50(\mathrm{~d}, J=7.2 \mathrm{~Hz}, 1 \mathrm{H}), 4.79$ (dd, $J=5.6$ and 2.8 $\mathrm{Hz}, 1 \mathrm{H}), 4.49-4.35$ (m, 2H), 3.67 (s, 3H), 3.06 (ddd, $J=17.6,14.0$ and $4.0 \mathrm{~Hz}, 2 \mathrm{H}) .{ }^{13} \mathrm{C}$ NMR (100 MHz, $\left.\mathrm{CDCl}_{3}\right) \delta 173.4,158.1,157.6,148.8,136.8,136.3,129.2,128.4,126.8$, 122.1, 122.0, 54.0, 52.1, 45.4, 38.4; IR (neat) $v=3748,1735,1629,1558,1247 \mathrm{~cm}^{-1}$; Anal. Calcd for $\mathrm{C}_{17} \mathrm{H}_{19} \mathrm{~N}_{3} \mathrm{O}_{3}$ : C, 65.16\%; H, 6.11\%; N, 13.41\%, found $\mathrm{C}, 65.03 \%, \mathrm{H}$, $6.08 \%, \mathrm{~N}, 13.40 \%$.

(3) Compound (S)-8: Yield: 97\%. ${ }^{1} \mathrm{H}$ NMR (400 MHz, MeOD-d $\left.)_{4}\right) \delta 8.82(\mathrm{~d}, J=6.0 \mathrm{~Hz}$, $1 \mathrm{H}), 8.48(\mathrm{t}, J=8.0 \mathrm{~Hz}, 1 \mathrm{H}), 8.02(\mathrm{~d}, J=8.0 \mathrm{~Hz}, 1 \mathrm{H}), 7.91(\mathrm{t}, J=6.8 \mathrm{~Hz}, 1 \mathrm{H}), 6.86-6.72$ (br, 2H), $4.75(\mathrm{~d}, J=6.4 \mathrm{~Hz}, 2 \mathrm{H}), 4.59$ (t, $J=8.0 \mathrm{~Hz}, 2 \mathrm{H}), 3.68$ (s, 3H), 2.00-1.90 (m, 2H), 1.77-1.67 (m, 1H), 1.62-1.42 (m, 4H), $0.99(\mathrm{t}, J=7.6,3 \mathrm{H}), 0.94(\mathrm{~d}, J=6.8 \mathrm{~Hz}, 3 \mathrm{H})$, $0.91(\mathrm{~d}, J=6.8 \mathrm{~Hz}, 3 \mathrm{H}) .{ }^{13} \mathrm{C}$ NMR $\left(100 \mathrm{MHz}, \mathrm{MeOD}-d_{4}\right) \delta 175.6,159.9,157.7,146.6$, $128.2,127.4,58.6,53.1,52.7,41.8,33.4,25.9,23.2,21.9,20.5,13.8$. IR (neat) $v=2960$, 
$1761,1706,1429 \mathrm{~cm}^{-1}$; The product was used for the next step directly without further characterization.

(4) Compound (S)-9: Yield: 92\%. ${ }^{1} \mathrm{H}$ NMR (400 MHz, MeOD- $\left.d_{4}\right) \delta 8.97$ (d, $J=6.0 \mathrm{~Hz}$, 1H), $8.47(\mathrm{t}, J=8.0 \mathrm{~Hz}, 1 \mathrm{H}), 7.94(\mathrm{t}, J=7.2 \mathrm{~Hz}, 1 \mathrm{H}), 7.86(\mathrm{~d}, J=8.0 \mathrm{~Hz}, 1 \mathrm{H}), 7.35-7.16$ (m, 5H), 4.70-4.53 (m, 5H), $3.68(\mathrm{~s}, 3 \mathrm{H}), 3.13(\mathrm{dd}, J=13.6$ and $5.6 \mathrm{~Hz}, 1 \mathrm{H}), 2.97$ (dd, $J$ $=13.6$ and $8.4 \mathrm{~Hz}, 1 \mathrm{H}), 2.0-1.86(\mathrm{~m}, 2 \mathrm{H}), 1.54-1.44(\mathrm{~m}, 2 \mathrm{H}), 0.99(\mathrm{t}, J=7.6 \mathrm{~Hz}, 3 \mathrm{H}) .{ }^{13} \mathrm{C}$ NMR (100 MHz, MeOD-d $\left.d_{4}\right) \delta 174.2,159.5,157.7,146.7,146.5,146.5,138.1,130.3$, $129.5,128.2,127.9,127.3,58.6,55.9,52.8,41.8,38.9,33.4,20.6,13.8$. IR (neat) $v=$ $1772,1716,1436 \mathrm{~cm}^{-1}$; The product was used for the next step directly without further characterization.

(5) Compound (S)-11a: Yield: 91\%. Brown solid; mp: $42-44^{\circ} \mathrm{C}$; $[\alpha]_{\mathrm{D}}{ }^{20}=-14.26^{\circ}(\mathrm{c}=$ 1.08, EtOH); ${ }^{1} \mathrm{H}$ NMR (400 MHz, MeOD- $\left.d_{4}\right) \delta 8.90(\mathrm{dd}, J=6.0$ and $1.2 \mathrm{~Hz}, 1 \mathrm{H}), 8.52$ (td, $J=8.0$ and $1.2 \mathrm{~Hz}, 1 \mathrm{H}), 8.06(\mathrm{~d}, J=8.0 \mathrm{~Hz}, 1 \mathrm{H}), 7.96-7.92(\mathrm{~m}, 1 \mathrm{H}), 4.85-4.74(\mathrm{~m}$, $2 \mathrm{H}), 4.63(\mathrm{t}, J=8.0 \mathrm{~Hz}, 2 \mathrm{H}), 4.35-4.25(\mathrm{~m}, 1 \mathrm{H}), 3.70(\mathrm{~s}, 3 \mathrm{H}), 2.05-1.92(\mathrm{~m}, 2 \mathrm{H}), 1.80-$ $1.69(\mathrm{~m}, 1 \mathrm{H}), 1.64-1.45(\mathrm{~m}, 4 \mathrm{H}), 1.03(\mathrm{t}, J=7.2 \mathrm{~Hz}, 3 \mathrm{H}), 0.96(\mathrm{~d}, J=6.8 \mathrm{~Hz}, 3 \mathrm{H}), 0.93$ $(\mathrm{d}, J=6.8 \mathrm{~Hz}, 3 \mathrm{H}) .{ }^{13} \mathrm{C}$ NMR $\left(100 \mathrm{MHz}, \mathrm{MeOD}-d_{4}\right) \delta 175.6,160.0,157.9,146.8,146.6$, $128.4,127.4,58.7,53.1,52.7,41.9,41.8,33.5,25.9,23.3,21.9,20.6,13.8$; IR (neat) $v=$ 3748, 2959, 1735, 1699, 1651, 1558, $1063 \mathrm{~cm}^{-1}$; HRMS (ESI +) m/z (\%) Calcd for $\left[\mathrm{C}_{18} \mathrm{H}_{30} \mathrm{~N}_{3} \mathrm{O}_{3}\right]^{+}: 336.2287$, found: 336.2287 .

(6) Compound (S)-11b: Yield: 75\%. Brown solid; mp: $82-84^{\circ} \mathrm{C} ;[\alpha]_{\mathrm{D}}{ }^{20}=-15.71^{\circ}(\mathrm{c}=$ 0.91, EtOH); ${ }^{1} \mathrm{H}$ NMR $\left(400 \mathrm{MHz}, \mathrm{MeOD}-d_{4}\right) \delta 8.87(\mathrm{~d}, J=6.4 \mathrm{~Hz}, 1 \mathrm{H}), 8.51(\mathrm{t}, J=7.6$ $\mathrm{Hz}, 1 \mathrm{H}), 8.05(\mathrm{~d}, J=8.0 \mathrm{~Hz}, 1 \mathrm{H}), 7.94(\mathrm{t}, J=6.8 \mathrm{~Hz}, 1 \mathrm{H}), 4.90-4.71(\mathrm{~m}, 2 \mathrm{H}), 4.61(\mathrm{t}, J=$ 7.6 Hz, 2H), 4.35-4.25 (m, 1H), $3.70(\mathrm{~s}, 3 \mathrm{H}), 2.04-1.92(\mathrm{~m}, 2 \mathrm{H}), 1.79-1.69(\mathrm{~m}, 1 \mathrm{H}), 1.66-$ 
$1.42(\mathrm{~m}, 4 \mathrm{H}), 1.02(\mathrm{t}, J=7.2 \mathrm{~Hz}, 3 \mathrm{H}), 0.96(\mathrm{~d}, J=6.4 \mathrm{~Hz}, 3 \mathrm{H}), 0.93(\mathrm{~d}, J=6.4 \mathrm{~Hz}, 3 \mathrm{H})$. ${ }^{13} \mathrm{C}$ NMR $\left(100 \mathrm{MHz}, \mathrm{MeOD}-d_{4}\right) \delta 175.6,160.1,157.9,146.7,146.6,128.3,127.4,58.6$, 53.1, 52.7, 41.9, 41.8, 33.5, 25.9, 23.3, 21.8, 20.6, 13.8; IR (neat) $v=3748,2961,1735$, 1624, 1541, $836 \mathrm{~cm}^{-1}$; HRMS (ESI + ) m/z (\%) Calcd for $\left[\mathrm{C}_{18} \mathrm{H}_{30} \mathrm{~N}_{3} \mathrm{O}_{3}\right]^{+}: 336.2287$, found: 336.2283; HRMS (ESI -) m/z (\%) Calcd for [ $\left[\mathrm{PF}_{6}\right]$ : 144.9642 , found: 144.9816.

(7) Compound (S)-11c: Yield: 77\%. Brown oil; $[\alpha]_{\mathrm{D}}{ }^{20}=-11.74^{\circ}(\mathrm{c}=0.92, \mathrm{EtOH}) ;{ }^{1} \mathrm{H}$ NMR (400 MHz, MeOD-d $) \delta 8.88(\mathrm{~d}, J=6.0 \mathrm{~Hz}, 1 \mathrm{H}), 8.51(\mathrm{t}, J=7.6 \mathrm{~Hz}, 1 \mathrm{H}), 8.05(\mathrm{~d}$, $J=8.4 \mathrm{~Hz}, 1 \mathrm{H}), 7.94(\mathrm{t}, J=7.2 \mathrm{~Hz}, 1 \mathrm{H}), 4.88-4.72(\mathrm{~m}, 2 \mathrm{H}), 4.62(\mathrm{t}, J=8.0 \mathrm{~Hz}, 2 \mathrm{H})$, 4.35-4.25 (m, 1H), $3.70(\mathrm{~s}, 3 \mathrm{H}), 2.05-1.92(\mathrm{~m}, 2 \mathrm{H}), 1.79-1.68(\mathrm{~m}, 1 \mathrm{H}), 1.65-1.44(\mathrm{~m}$, 4H), $1.02(\mathrm{t}, J=7.2 \mathrm{~Hz}, 3 \mathrm{H}), 0.96(\mathrm{~d}, J=6.8 \mathrm{~Hz}, 3 \mathrm{H}), 0.93(\mathrm{~d}, J=6.8 \mathrm{~Hz}, 3 \mathrm{H}) .{ }^{13} \mathrm{C} \mathrm{NMR}$ $\left(100 \mathrm{MHz}, \mathrm{MeOD}-d_{4}\right) \delta 175.6,160.0,157.9,146.8,146.6,128.3,127.4,121.1(\mathrm{q}, J=$ $319.2 \mathrm{~Hz}), 58.7,53.1,52.7,41.9,41.8,33.5,25.9,23.2,21.8,20.6,13.8$; IR (neat) $v=$ 3748, 1734, 1699, 1651, 1558, 1189, $616 \mathrm{~cm}^{-1}$; HRMS $($ ESI +$) \mathrm{m} / \mathrm{z}(\%)$ Calcd for $\left[\mathrm{C}_{18} \mathrm{H}_{30} \mathrm{~N}_{3} \mathrm{O}_{3}\right]^{+}:$336.2287, found: 336.2284; HRMS (ESI -) $\mathrm{m} / \mathrm{z}$ (\%) Calcd for [N( $\left.\left(\mathrm{SO}_{2} \mathrm{CF}_{3}\right)_{2}\right]: 279.9173$, found: 279.9209 .

(8) Compound (S)-12a: Yield: 97\%. Yellow solid; mp: $48-50^{\circ} \mathrm{C} ;[\alpha]_{\mathrm{D}}^{20}=3.14^{\circ}(\mathrm{c}=$ 0.35, EtOH); ${ }^{1} \mathrm{H}$ NMR $\left(400 \mathrm{MHz}, \mathrm{MeOD}-d_{4}\right) \delta 9.01(\mathrm{~d}, J=6.0 \mathrm{~Hz}, 1 \mathrm{H}), 8.58(\mathrm{t}, J=8.0$ Hz, 1H), 8.05 (t, $J=6.4 \mathrm{~Hz}, 1 \mathrm{H}), 7.97(\mathrm{~d}, J=8.0 \mathrm{~Hz}, 1 \mathrm{H}), 7.50-7.28(\mathrm{~m}, 5 \mathrm{H}), 4.83$ (s, $2 \mathrm{H}), 4.75-4.65(\mathrm{~m}, 3 \mathrm{H}), 3.82(\mathrm{~s}, 3 \mathrm{H}), 3.27(\mathrm{dd}, J=14.0$ and $5.2 \mathrm{~Hz}, 1 \mathrm{H}), 3.08(\mathrm{dd}, J=$ 14.0 and $4.8 \mathrm{~Hz}, 1 \mathrm{H}), 2.10-1.90(\mathrm{~m}, 2 \mathrm{H}), 1.67-1.54(\mathrm{~m}, 2 \mathrm{H}), 1.21(\mathrm{t}, J=7.6 \mathrm{~Hz}, 3 \mathrm{H}) .{ }^{13} \mathrm{C}$ NMR (100 MHz, MeOD-d $\left.d_{4}\right) \delta 174.3,159.6,157.9,146.8,146.6,138.2,130.4,129.6$, $128.2,127.9,127.4,58.7,55.9,52.7,41.8,39.0,33.5,20.6,13.8 ;$ IR (neat) $v=3748$, 2959, 1735, 1651, $1558 \mathrm{~cm}^{-1}$; HRMS (ESI +) m/z (\%) Calcd for $\left[\mathrm{C}_{21} \mathrm{H}_{28} \mathrm{~N}_{3} \mathrm{O}_{3}\right]^{+}$: 
370.2131, found: 370.2126; HRMS (ESI -) m/z (\%) Calcd for [BF $]_{4}^{-}: 87.0029$, found: 87.0044 .

(9) Compound (S)-12b: Yield: 78\%. Yellow solid; mp: $50-52^{\circ} \mathrm{C} ;[\alpha]_{\mathrm{D}}{ }^{20}=2.35^{\circ}(\mathrm{c}=$ 0.34, EtOH); ${ }^{1} \mathrm{H}$ NMR (400 MHz, MeOD- $\left.d_{4}\right) \delta 8.84(\mathrm{~d}, J=6.0 \mathrm{~Hz}, 1 \mathrm{H}), 8.45(\mathrm{t}, J=8.0$ Hz, 1H), $7.92(\mathrm{t}, J=6.8 \mathrm{~Hz}, 1 \mathrm{H}), 7.84(\mathrm{~d}, J=8.0 \mathrm{~Hz}, 1 \mathrm{H}), 7.38-7.14(\mathrm{~m}, 5 \mathrm{H}), 4.69$ (s, $2 \mathrm{H}), 4.63-4.53(\mathrm{~m}, 3 \mathrm{H}), 3.70(\mathrm{~s}, 3 \mathrm{H}), 3.15(\mathrm{dd}, J=13.6$ and $5.2 \mathrm{~Hz}, 1 \mathrm{H}), 2.96(\mathrm{dd}, J=$ 13.6 and $8.8 \mathrm{~Hz}, 1 \mathrm{H}), 2.00-1.85(\mathrm{~m}, 2 \mathrm{H}), 1.55-1.40(\mathrm{~m}, 2 \mathrm{H}), 1.00(\mathrm{t}, J=7.6 \mathrm{~Hz}, 3 \mathrm{H}) .{ }^{13} \mathrm{C}$ NMR (100 MHz, MeOD-d $\left.d_{4}\right) \delta 174.3,159.6,157.8,146.7,146.6,138.1,130.4,129.6$, $128.1,127.9,127.3,58.6,55.9,52.7,41.7,39.0,33.4,20.6,13.8 ;$ IR (neat) $v=3748$, 2962, 1733, 1650, 1558, $836 \mathrm{~cm}^{-1}$; HRMS (ESI +) m/z (\%) Calcd for $\left[\mathrm{C}_{21} \mathrm{H}_{28} \mathrm{~N}_{3} \mathrm{O}_{3}\right]^{+}$: 370.2131, found: 370.2128; HRMS (ESI -) m/z (\%) Calcd for [PF $\left.{ }_{6}\right]^{\circ}: 144.9642$, found: 144.9717.

(10) Compound (S)-12c: Yield: $90 \%$. Yellow oil; $[\alpha]_{\mathrm{D}}{ }^{20}=2.2^{\circ}(\mathrm{c}=0.81, \mathrm{EtOH}) ;{ }^{1} \mathrm{H}$ NMR (400 MHz, MeOD-d $\left.{ }_{4}\right) \delta 8.84(\mathrm{~d}, J=6.0 \mathrm{~Hz}, 1 \mathrm{H}), 8.44(\mathrm{t}, J=8.0 \mathrm{~Hz}, 1 \mathrm{H}), 7.90$ (t, $J$ $=6.4 \mathrm{~Hz}, 1 \mathrm{H}), 7.82(\mathrm{~d}, J=8.0 \mathrm{~Hz}, 1 \mathrm{H}), 7.33-7.13(\mathrm{~m}, 5 \mathrm{H}), 4.68(\mathrm{~s}, 2 \mathrm{H}), 4.6-4.47(\mathrm{~m}, 3 \mathrm{H})$, $3.67(\mathrm{~s}, 3 \mathrm{H}), 3.14(\mathrm{dd}, J=14.0$ and $5.2 \mathrm{~Hz}, 1 \mathrm{H}), 2.95(\mathrm{dd}, J=13.6$ and $8.4 \mathrm{~Hz}, 1 \mathrm{H}), 1.97-$ $1.84(\mathrm{~m}, 2 \mathrm{H}), 1.52-1.39(\mathrm{~m}, 2 \mathrm{H}), 0.99$ (t, $J=7.2 \mathrm{~Hz}, 3 \mathrm{H}) .{ }^{13} \mathrm{C}$ NMR $(100 \mathrm{MHz}, \mathrm{MeOD}-$ $\left.d_{4}\right) \delta 174.2,159.6,157.8,146.7,146.6,138.1,130.3,129.5,128.1,127.9,127.4,121.1(\mathrm{q}$, $J=319.2 \mathrm{~Hz}$ ), 58.6, 55.8, 52.7, 41.7, 38.9, 33.5, 20.5, 13.8; IR (neat) $v=3748,1734$, 1696, 1650, 1558, 1189, $615 \mathrm{~cm}^{-1}$; HRMS (ESI +) m/z (\%) Calcd for $\left[\mathrm{C}_{21} \mathrm{H}_{28} \mathrm{~N}_{3} \mathrm{O}_{3}\right]^{+}$:

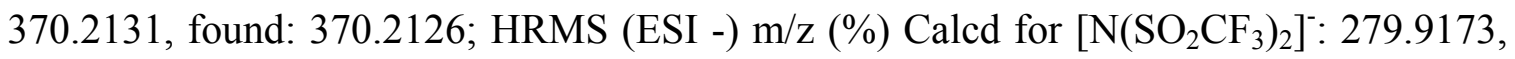
found: 279.9238 . 
z-1-18

Automation directory: /export/home/buni/vnarsys/data/auto_2006.03.03

File: exp trost
Sample id : tmpstudy
Sample: $z-1-18$

Pulse Sequence: s2pu

Solvent: edcl 13
Temp. $25.0 \mathrm{C} / 298.1 \mathrm{~K}$

Operator: buni
VWMRS-400 "nmr $400 "$

Relax. delay $1.000 \mathrm{sec}$
pulse 45 . 0 degrees
Acg. time $2.049 \mathrm{sec}$

widit $6410.3 \mathrm{~Hz}$

OBSERVE H1, $399.7386892 \mathrm{MH}$

DATA PROCESSING $0.2 \mathrm{~Hz}$

LT Ine broadenting $0.2 \mathrm{~Hz}$
FT

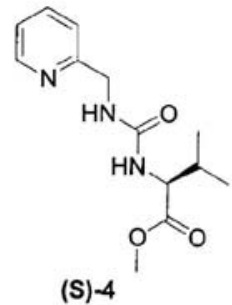

(S)-4

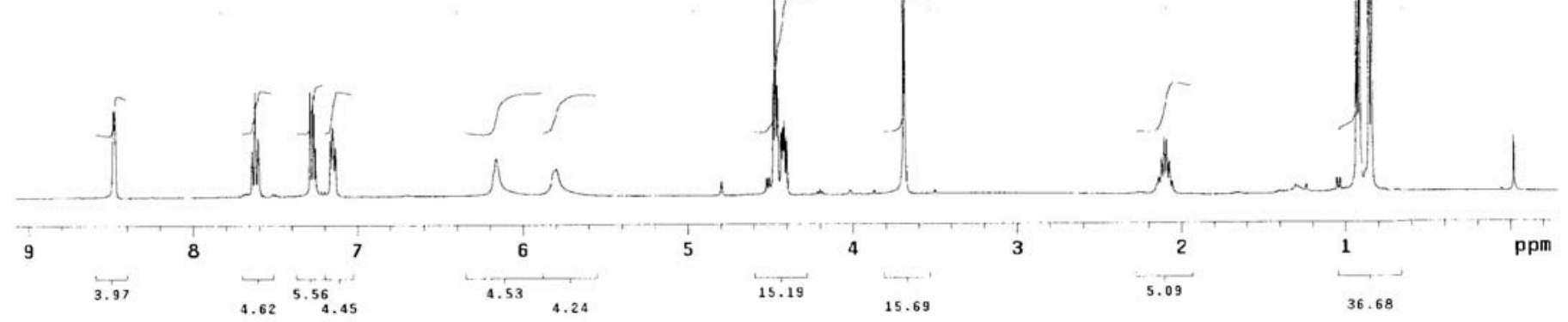


$2-1-18$

Autoaat ion directory: /export/home/buni/vnmrsys/data/auto_2006.03.03

Preaple exp: topstudy
sanple: $2-1-18$

Pulse Sequence: s2pul

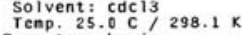

Oeerator: bunt
vNMRS 400 "nar 400

Relax. delay 1.000 sec
Pulse 45.0 dearees

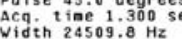

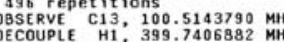

Power $38 \mathrm{~dB}$ '

cont inuously on
val T-1 16
nodu lated

DATA processing $0.5 \mathrm{~Hz}$

FT size $65536 \mathrm{hr}, 24 \mathrm{~min}, 54 \mathrm{sec}$
Total time $6 \mathrm{hr}$

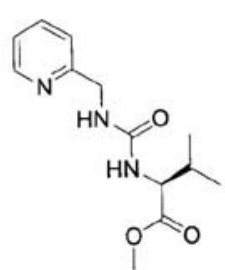

(S) -4

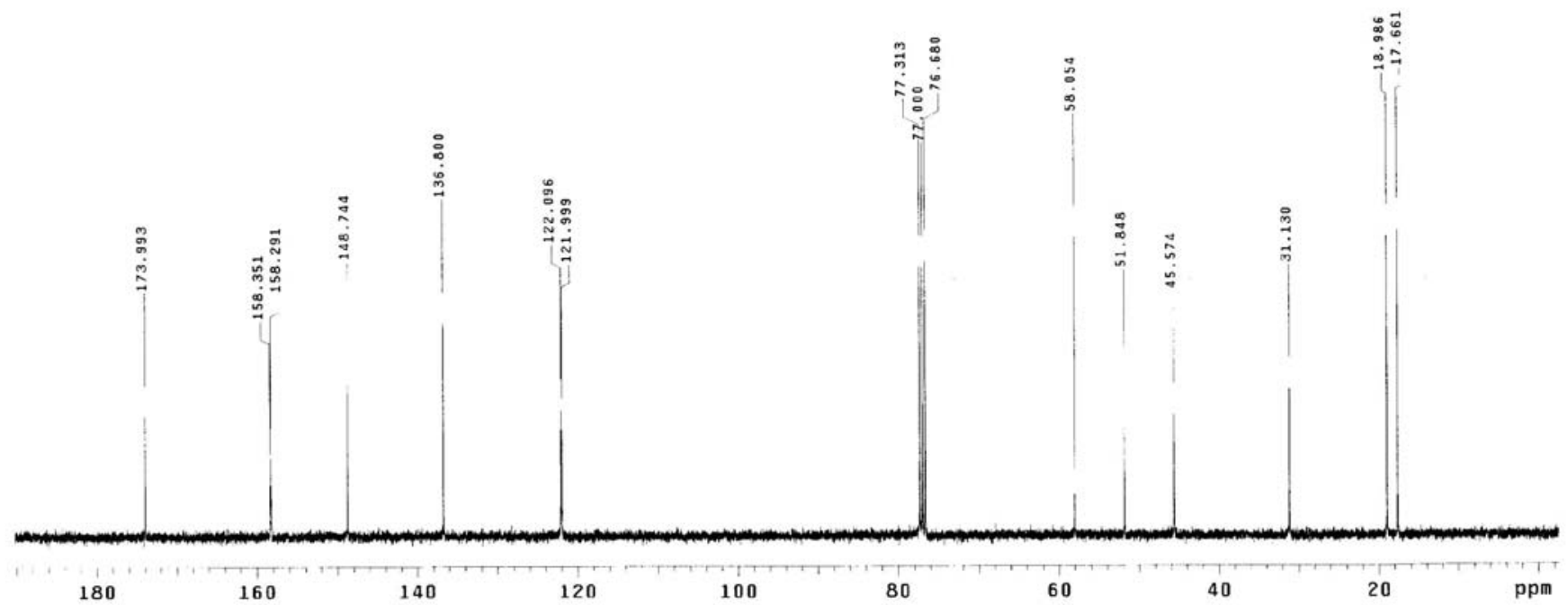


z-1-5s-1

Autonat ion directory? /export/hoae/buni/vnarsys/tata/auto 2006.06.22

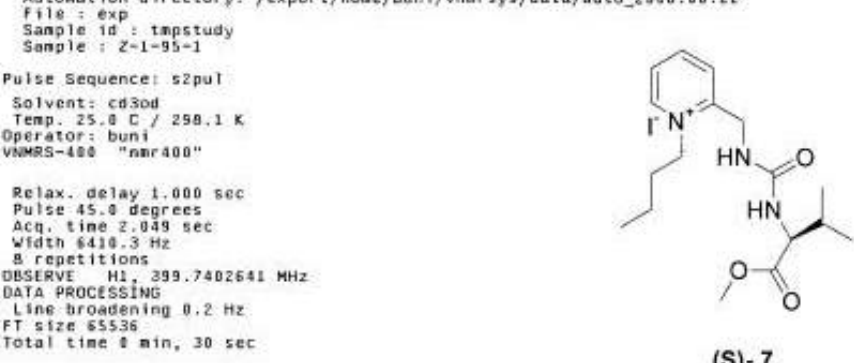

(S) -7

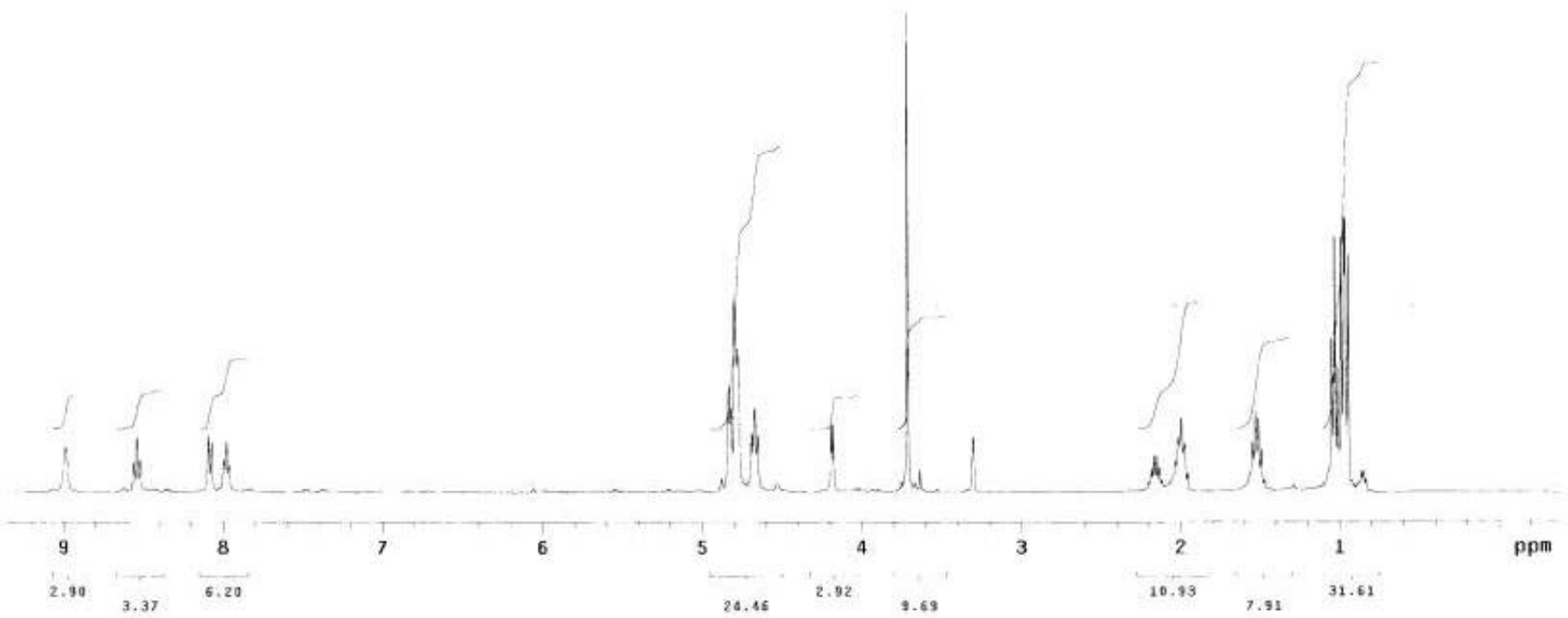


$2-1-95-1$

Autonation directory, /expers/hane/munt/4norsyi/sata/auto_ze06.06.22

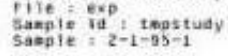
polse sequence: szpul Folvent: casou 250.1

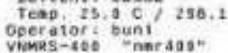

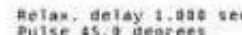

Acy in $20509.8 \mathrm{~Hz}$

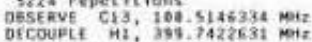

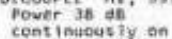

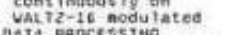

titsize besening $0.5 \mathrm{~Hz}$

Fotal ther $\mathrm{E} \mathrm{hr}, 24 \mathrm{~min}, \mathrm{~s} 4 \mathrm{se}$

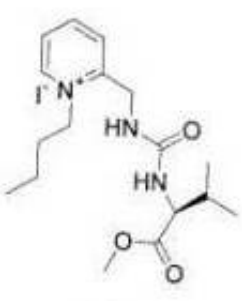

(S) 7

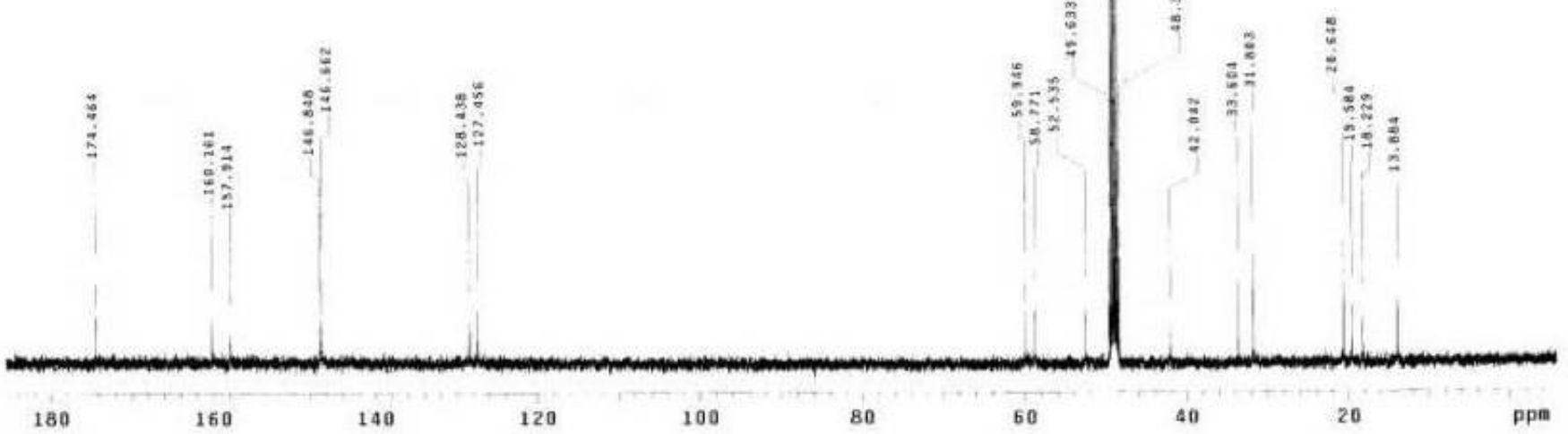


Autonat ton directory: /export/hoge/buni/vnmrsys/data/auto_2006.03.03

Sample: : $2-1-51$

Pulse Sequence: S2pul
Solvent: cd 3 od

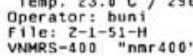

Relax. delay $1.000 \mathrm{sec}$
Pulse 45.0 degrees

Acat tine 2.049 se
Vidith $6410.3 \mathrm{~Hz}$

OBSERVE H1: 399.7402641 MHZ

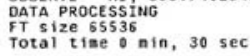

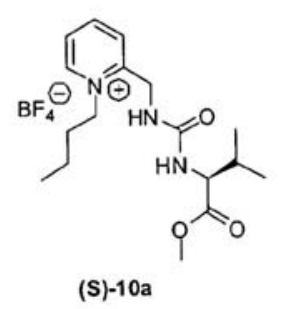

(S)-10a

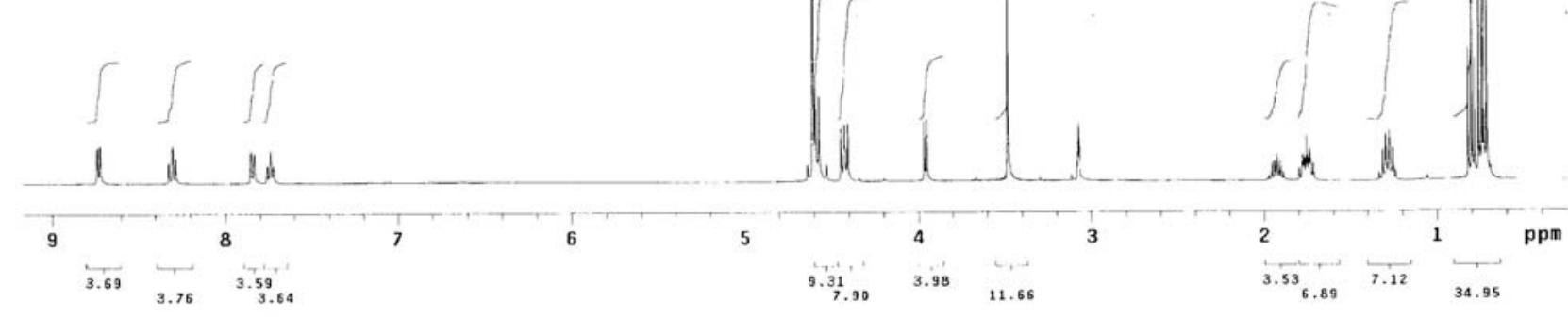


Automation directory: /export/hone/buni/vnar sys/data/auto_2006.03.03 Sample 10 in timpstudy

Pulse Sequence: $\$ 2$ pul

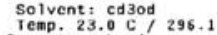

Operator: buni
vNMRS-400 "nar $400 \%$

Relax. delay $1,000 \mathrm{sec}$
Pulse 45.0 degrees

Acg time $1.300 \mathrm{sec}$
wifti $24509.8 \mathrm{~Hz}$

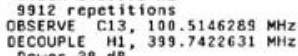

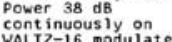

DATA PROCESSING

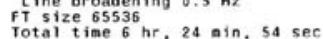
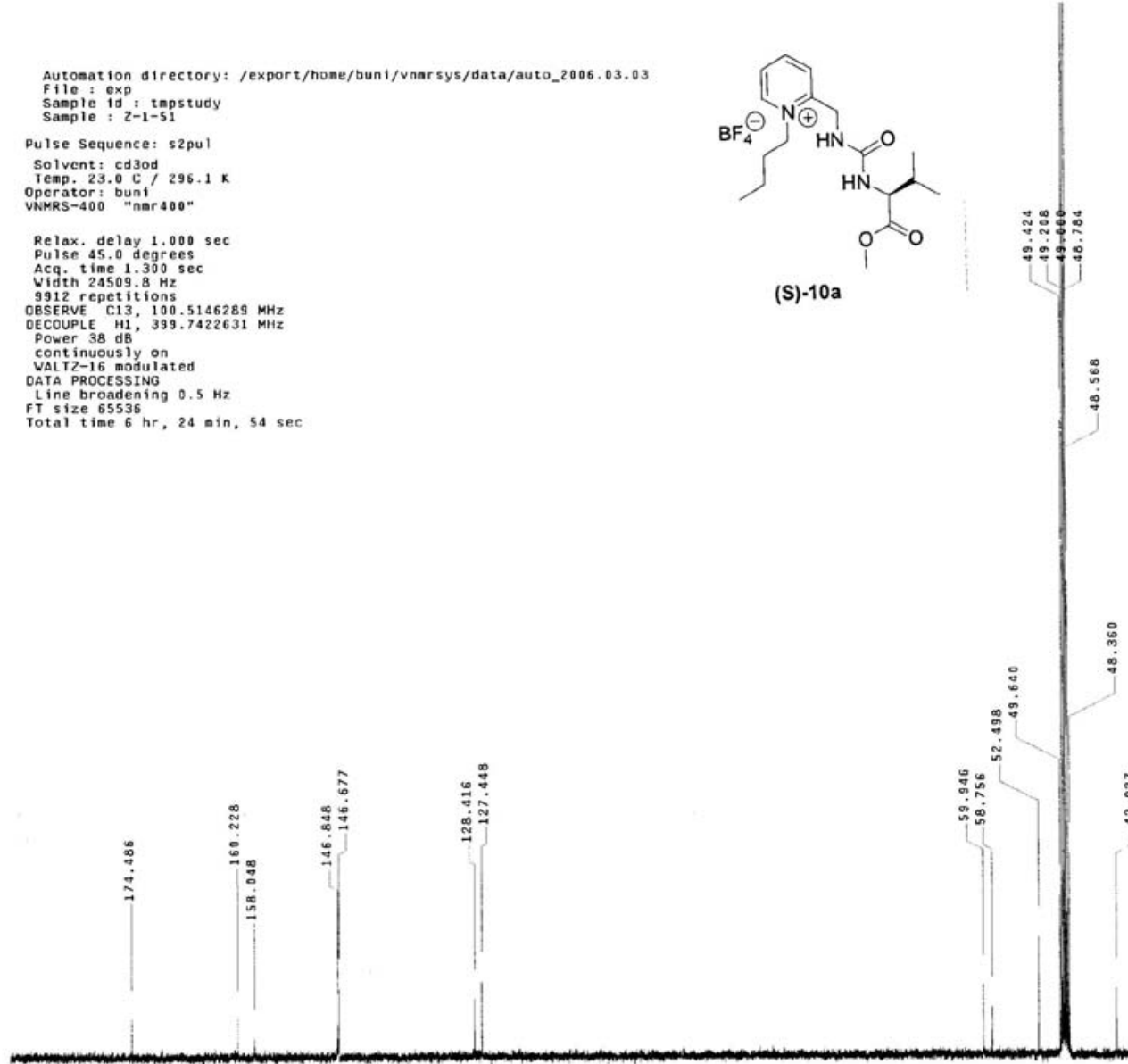

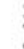

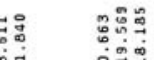

计

$180 \quad 160$

140

120

100

80

60

40

20

ppm 
2-1-38

Autoaation directory: /export/hong/buni/vnarsys/data/auto_2006.03.03

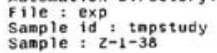

Pulse sequence: 52 pu

Solvent: cd3od $298.1 \times$

Operator: buni
VNMRS -400 -

Relax. delay 1.000
Pulse 45.0 degrees

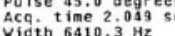

8 repetitions
OBSERVE H1, 399.7402641 MHZ

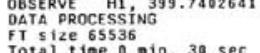

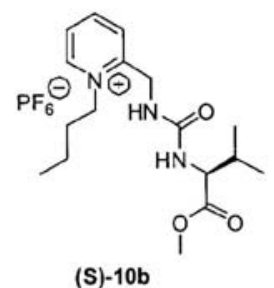


$2-1-38$

airectory:/export//ione/buni/ynarsys/data/auto_2006.03.03 File exp tomptud
sample id
Sample : $2-1-38$

Pulse sequence: 52 pu 1

Solvent:
Teap
Temp.

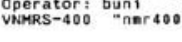

Relax. delay $1.000 \mathrm{sec}$
Pulse as. degrees

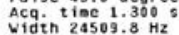

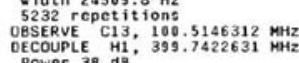

Power 38 ds
continuously on
WaATz-15 molulated

DATA PROCESSING

Fis size 65535 .
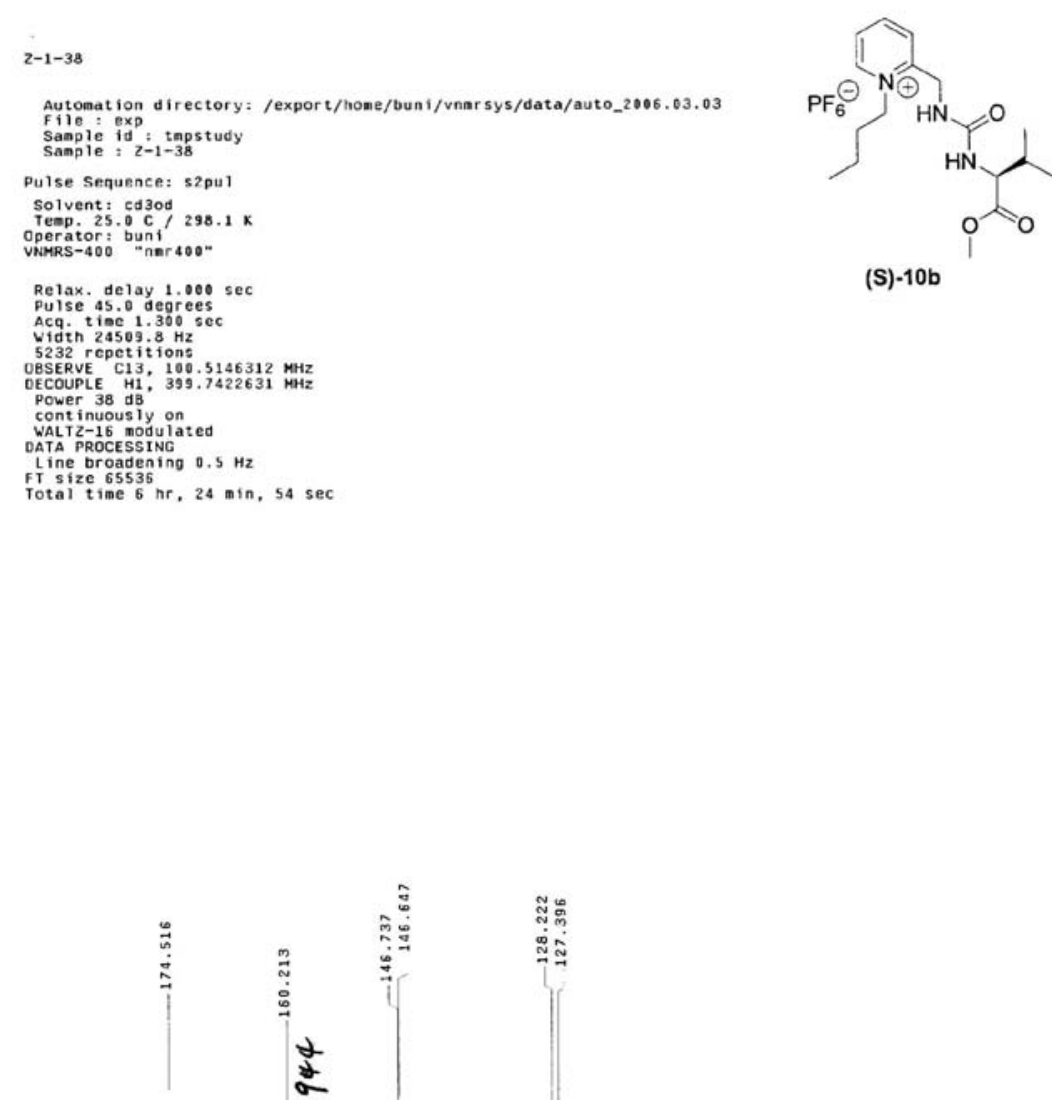

(S)-10b
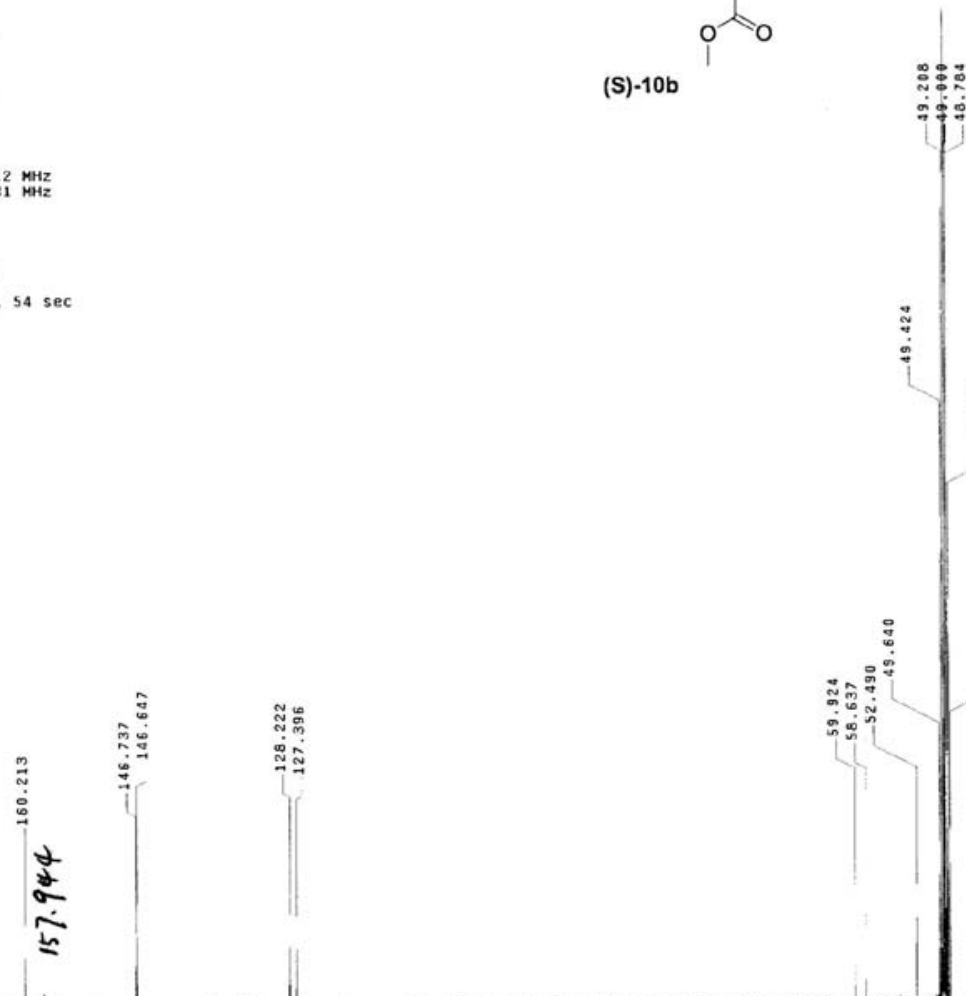

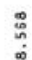

j)

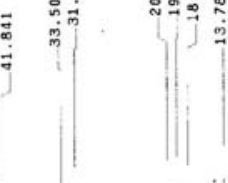

180

160

140

120

100

80

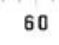

40

20 


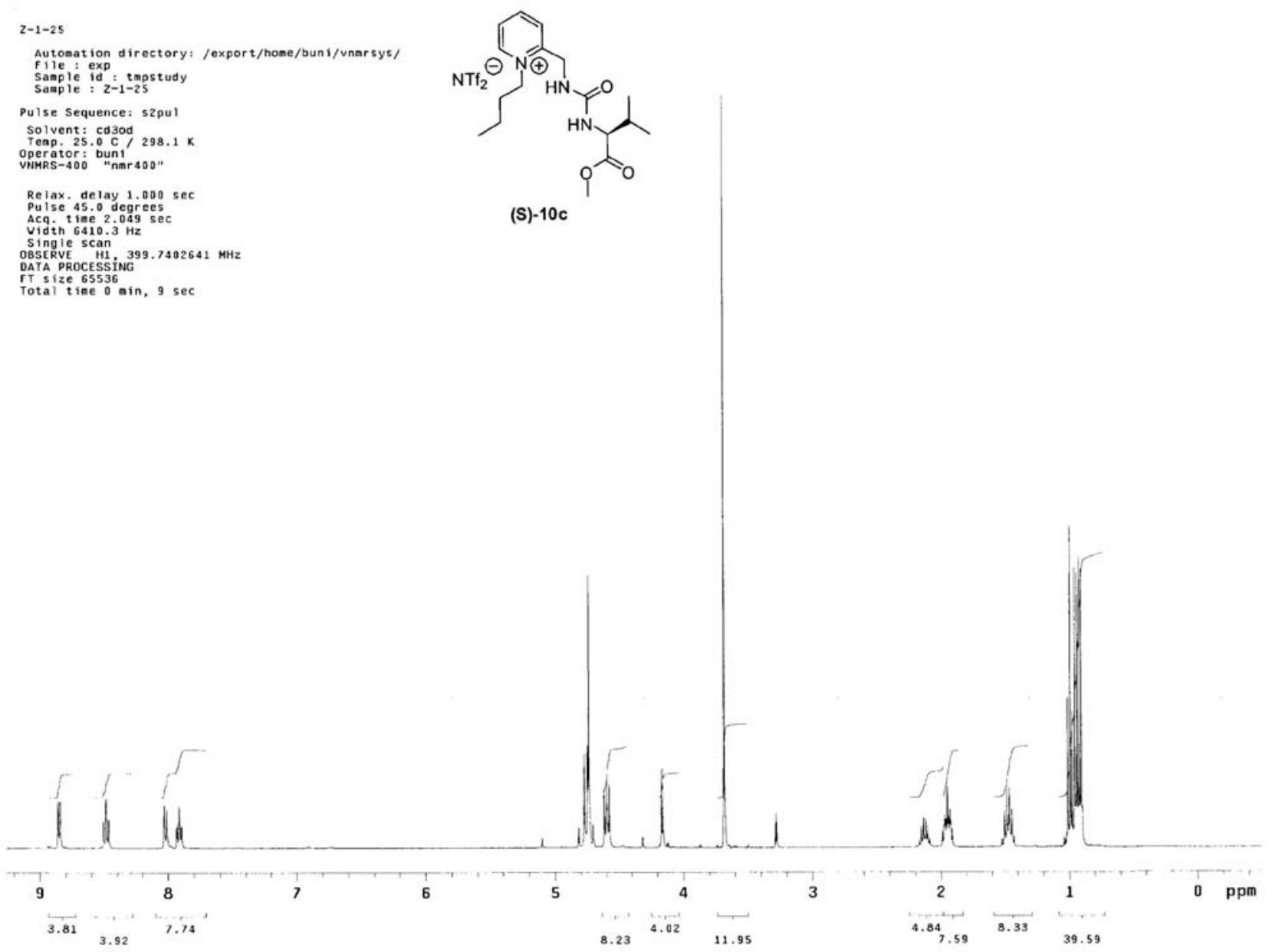




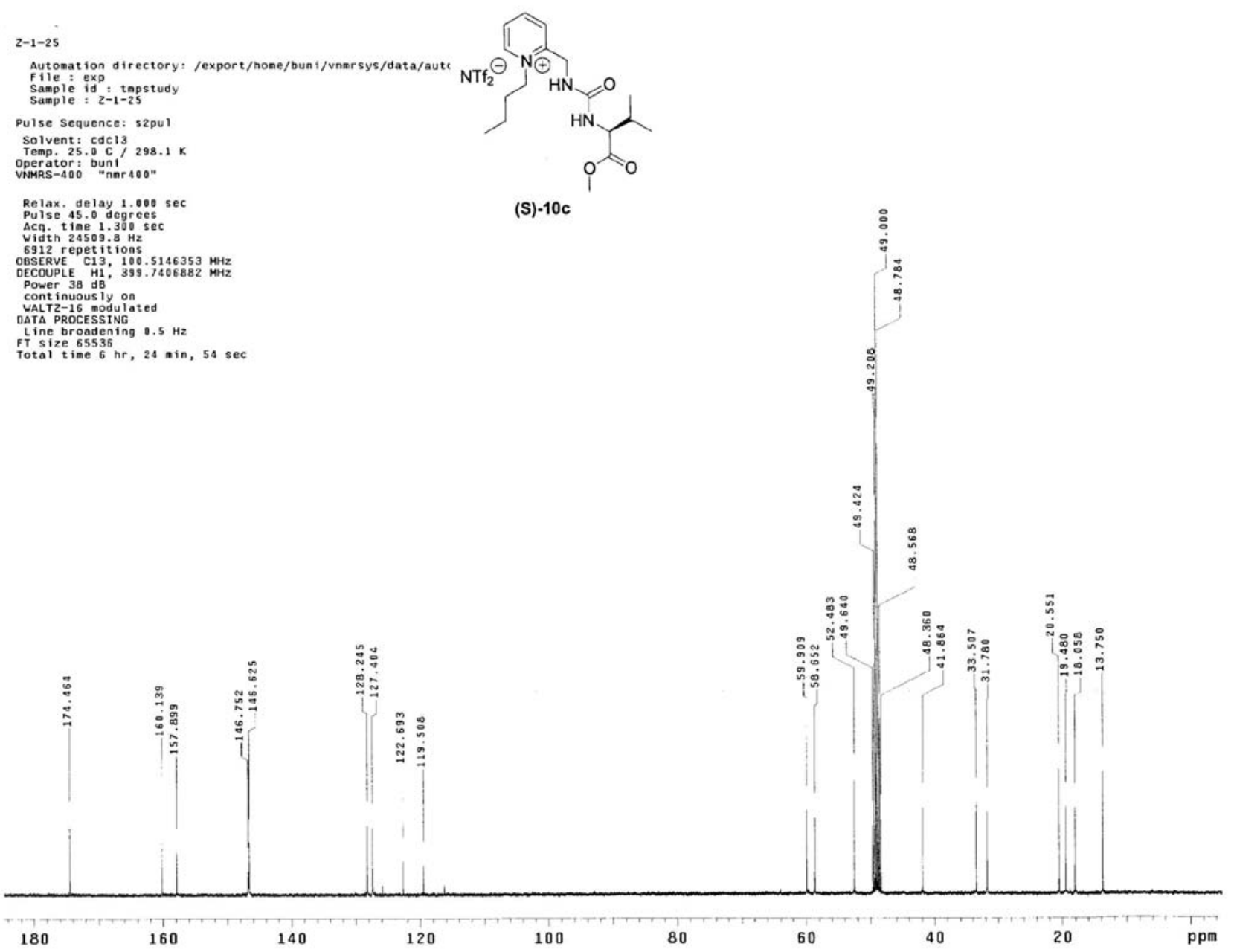




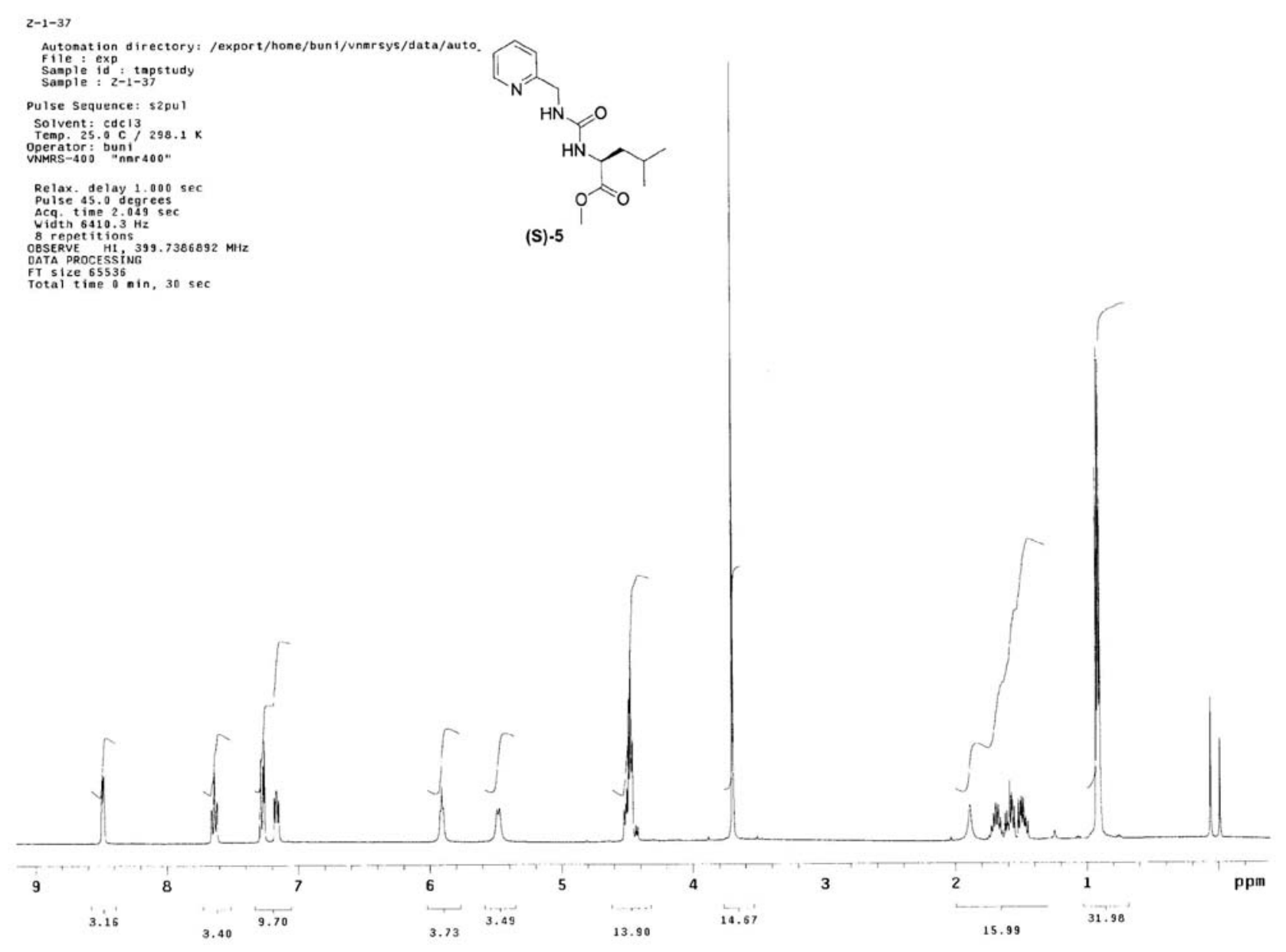

S16 
2-1-37

Automat ion directory: /export/home/buni/vnmersys/data/auto_2006.03.03

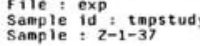

Pulse Sequence: 52 pul

Solvent: cdc13 13 .

Operator: buni
vWMRS -400 "nman $400 "$

Relax. delay $1.000 \mathrm{sec}$
Pulse 45.0 degrees

0
6

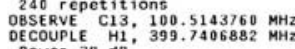

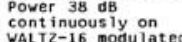

DATA Processinc

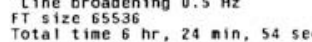

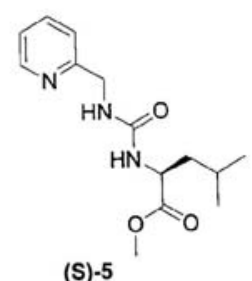

(S)-5

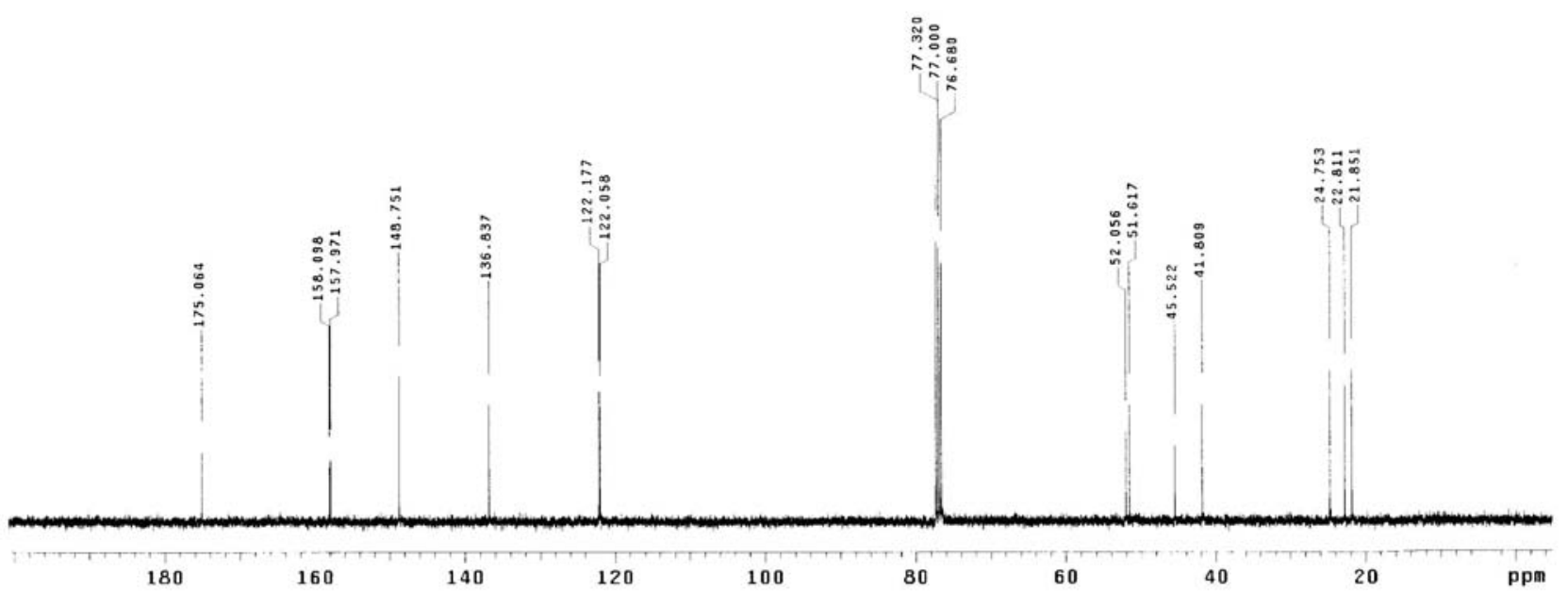


$z-1-9 x$

Autonat ion difectory: /export/hone/bun i/vnnes sys/data/auto_z006,06.22

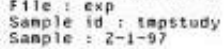

Pulse sequence: 82001

Solvent: cdjod
Teas. $26.0 \mathrm{C} / 289.1$

Operator: bunt
vimgs-400 "narapo"

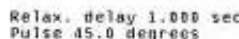

Acq. time 2.049 sec.

idin $540,3 \mathrm{Mz}$
feperitions

OESERVE H1 $399.7404915 \mathrm{MHz}$

tine broostenting $0.2 \mathrm{~Hz}$

FT 5 ize 65556
Total time 0 ain, 30 sed
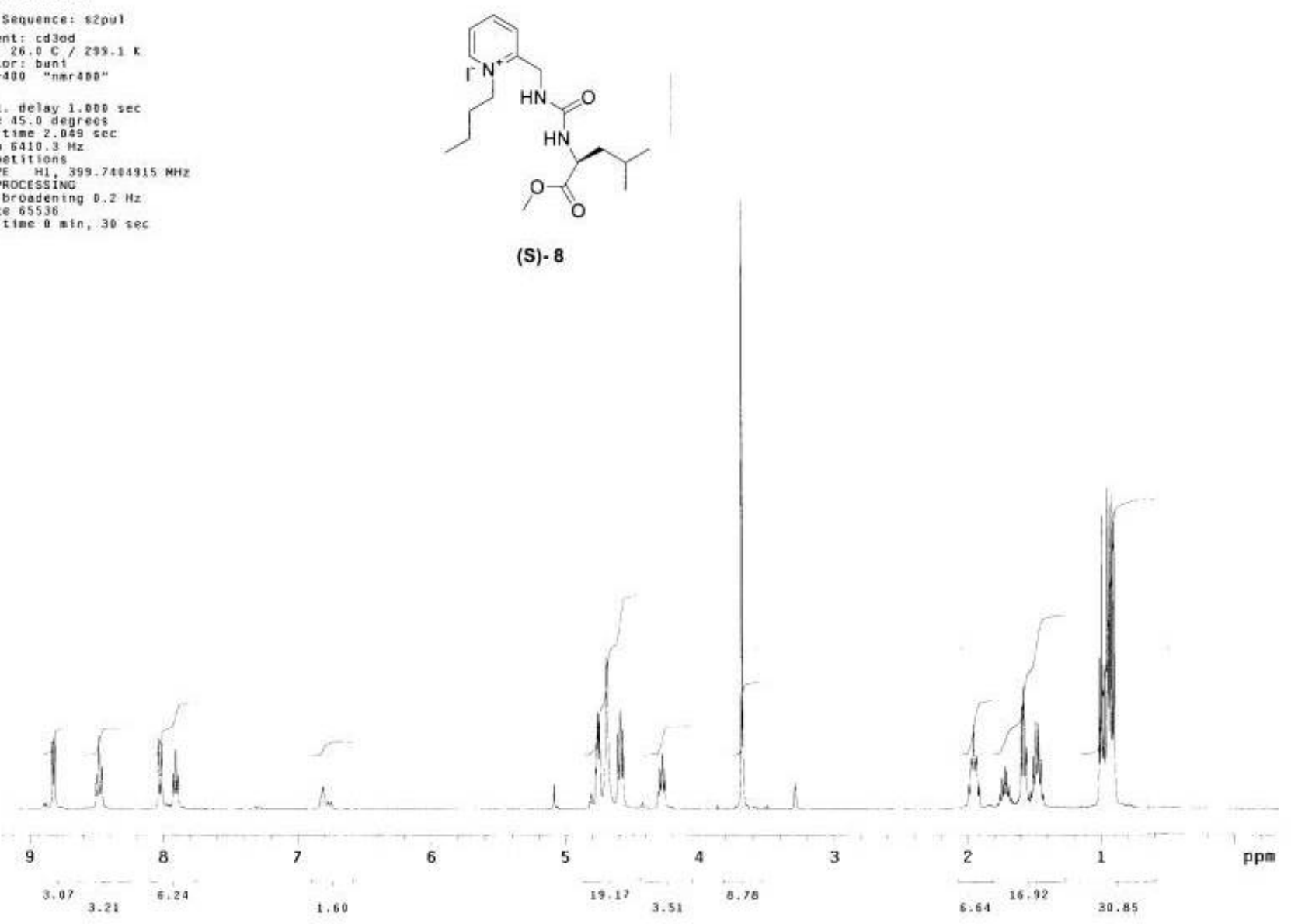
Autonation dírectory! /export/mone/bunt/vnar sys/data/auto_2006.96.22

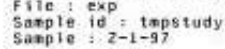

Pulse sequence: $52 \mathrm{pu}$

Solvent: cosod 299.1

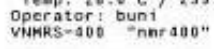

Relax. delay 1,000 sec
Pulse as.

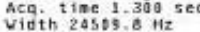

vidth 24509 . $8 \mathrm{~Hz}$
1848 repetitions

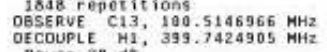

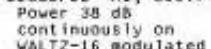

DATA PROCESTHS

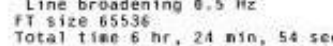

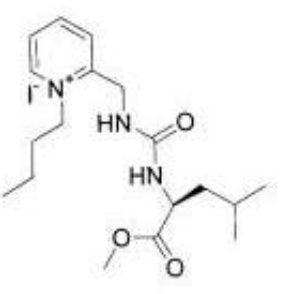

(S)- 8

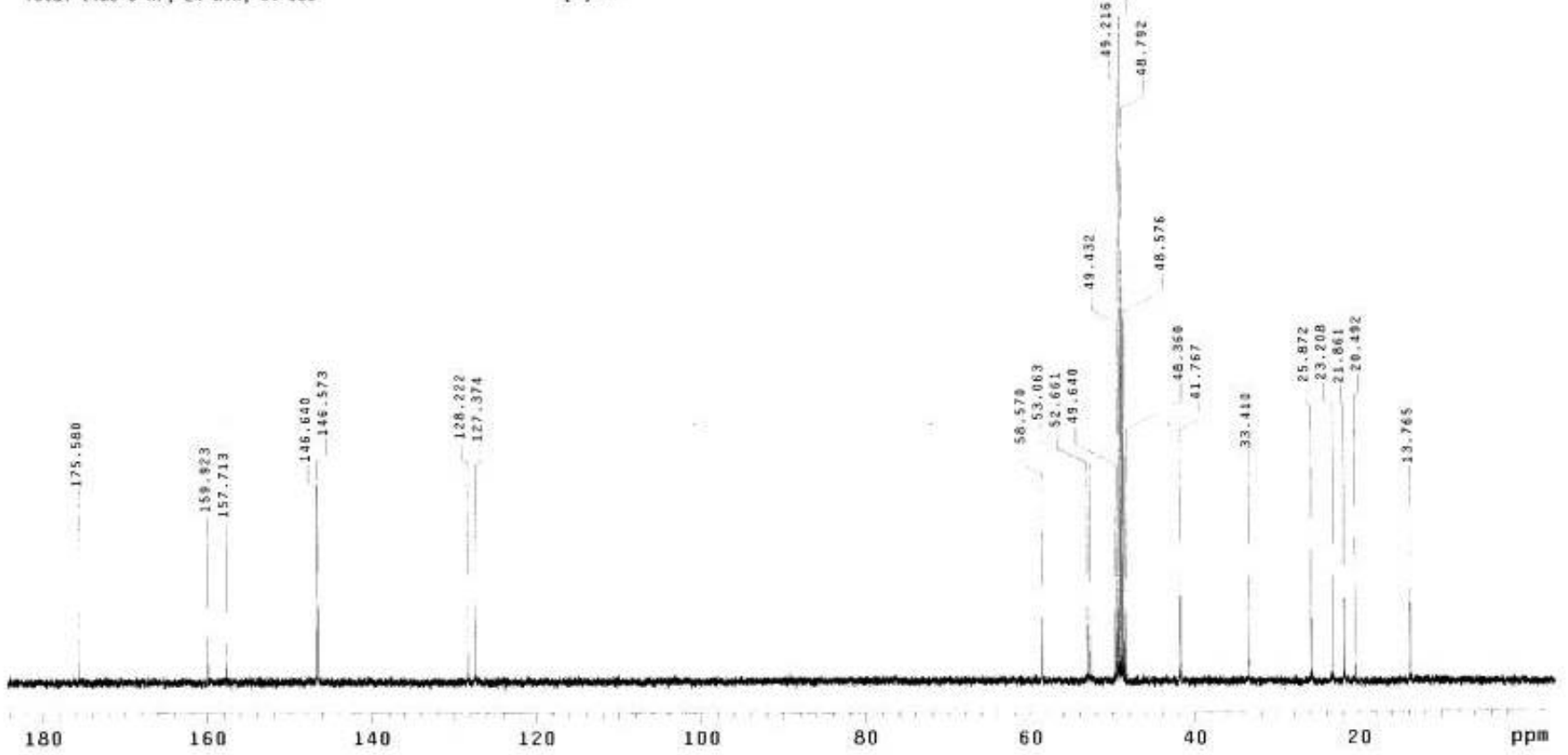


2-1-43

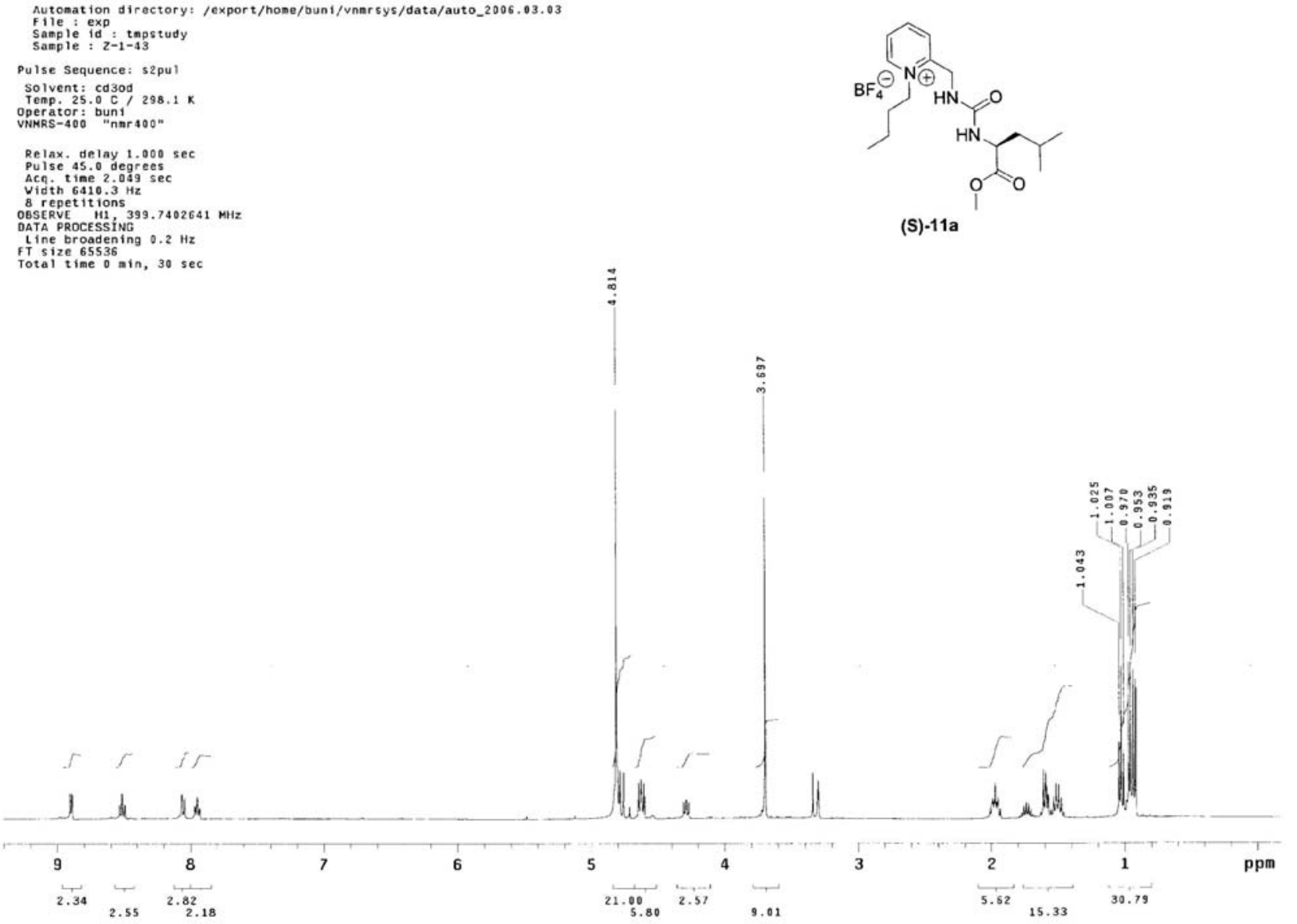


Automation directory: /export/home/buni/vnersys/data/auto_2006.03.03 File: exp
Sample id topstudy
Sample:

Pulse Sequence: s2pur
Solvent: cd3od 298.1

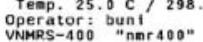

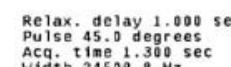

1944 repetitions
OBSERE C13, $100.5146327 \mathrm{MH}$
OECOUPLE H1 $399.7422631 \mathrm{MH}$

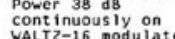

FT 512 . 65536
Total time $6 \mathrm{hr}, 24 \mathrm{~min}, 54 \mathrm{sec}$
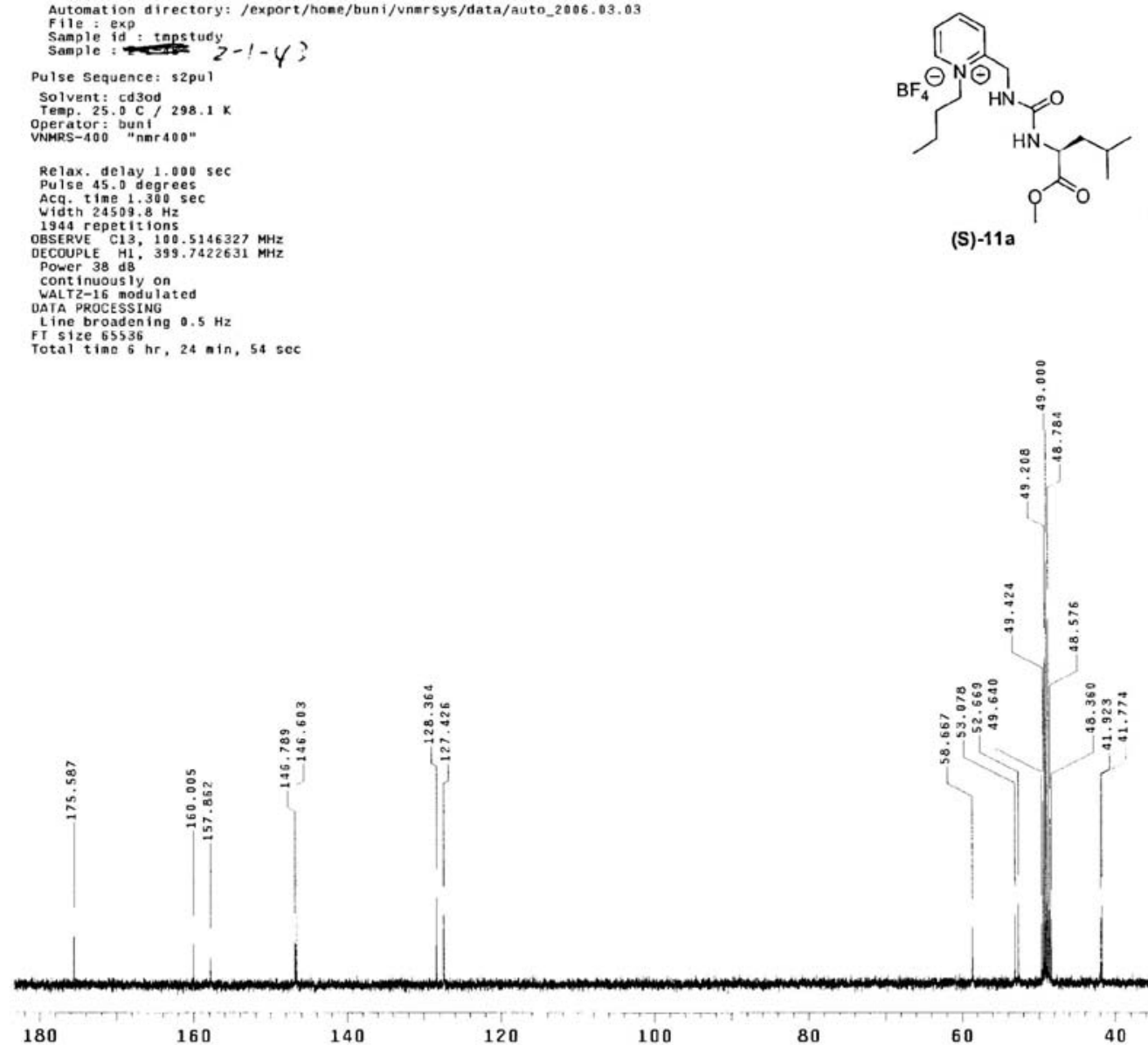

160

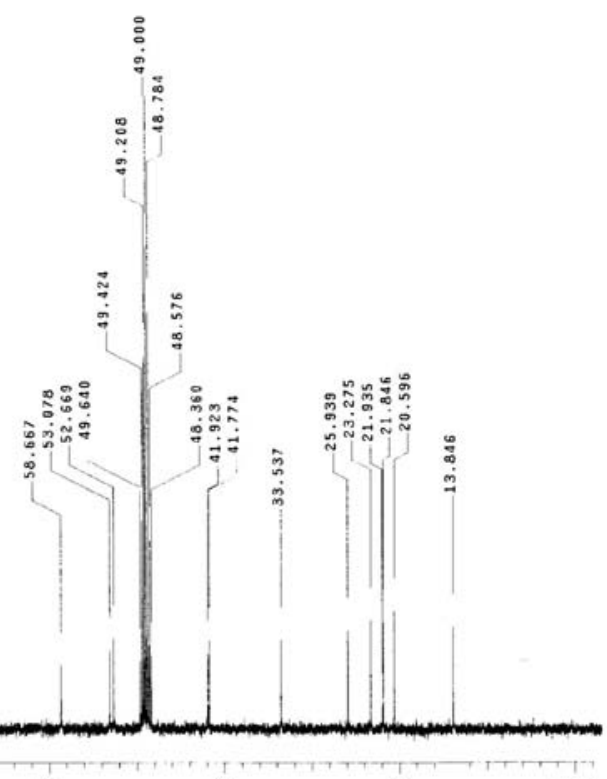


2-1-42

Automation directory: /export/home/bunt/vnarsys/data/auto_2006.03.03

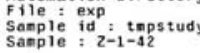

Pulse Sequence: s2pul

Solvent: cd3od $298.1 \times$
Teap. $25.0 \mathrm{C} / 298 \mathrm{x}$

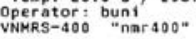

Relax. delay 1.000 sec
Pulse 45.0 degrees

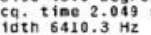

OSSERE H1 H1 399.7402641 MHZ

OATA PROCESSING
fT Size 65536 S
Total time 0 min, $30 \mathrm{sec}$
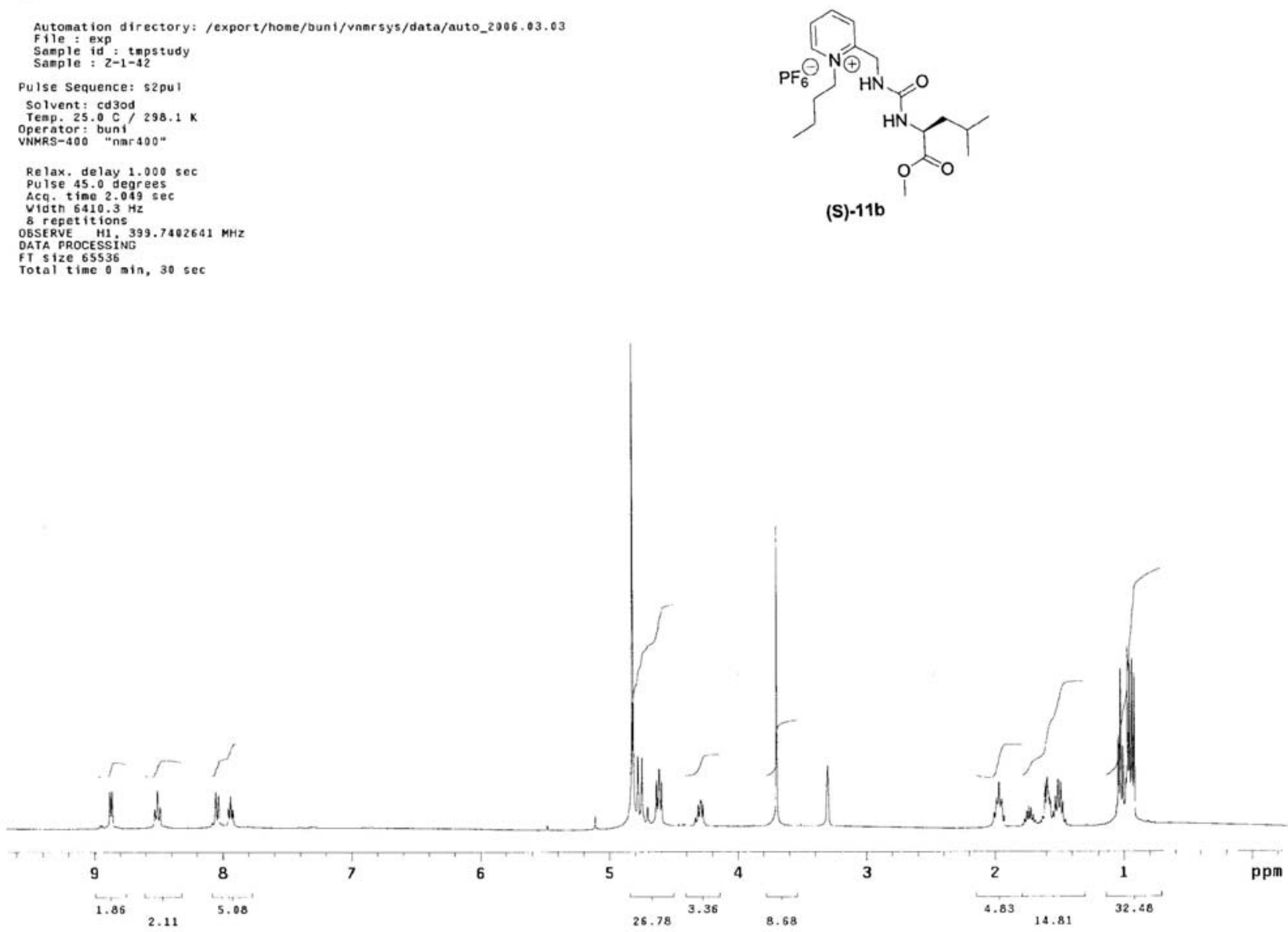
2-1-42

Automat ion directory: /export/home/bun 1/vnersys/data/auto_2006.03.03

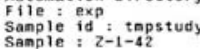

Pulse seq

cence: 52 pul

Tenp $25.0 \mathrm{C} / 298.1 \mathrm{~K}$
Tperator: buns

Relax. delay 1.000 sec

Acq. Time 11.300
actin $24509.8 \mathrm{~Hz}$
is
is

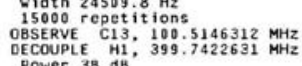

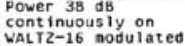

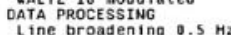

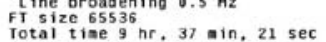

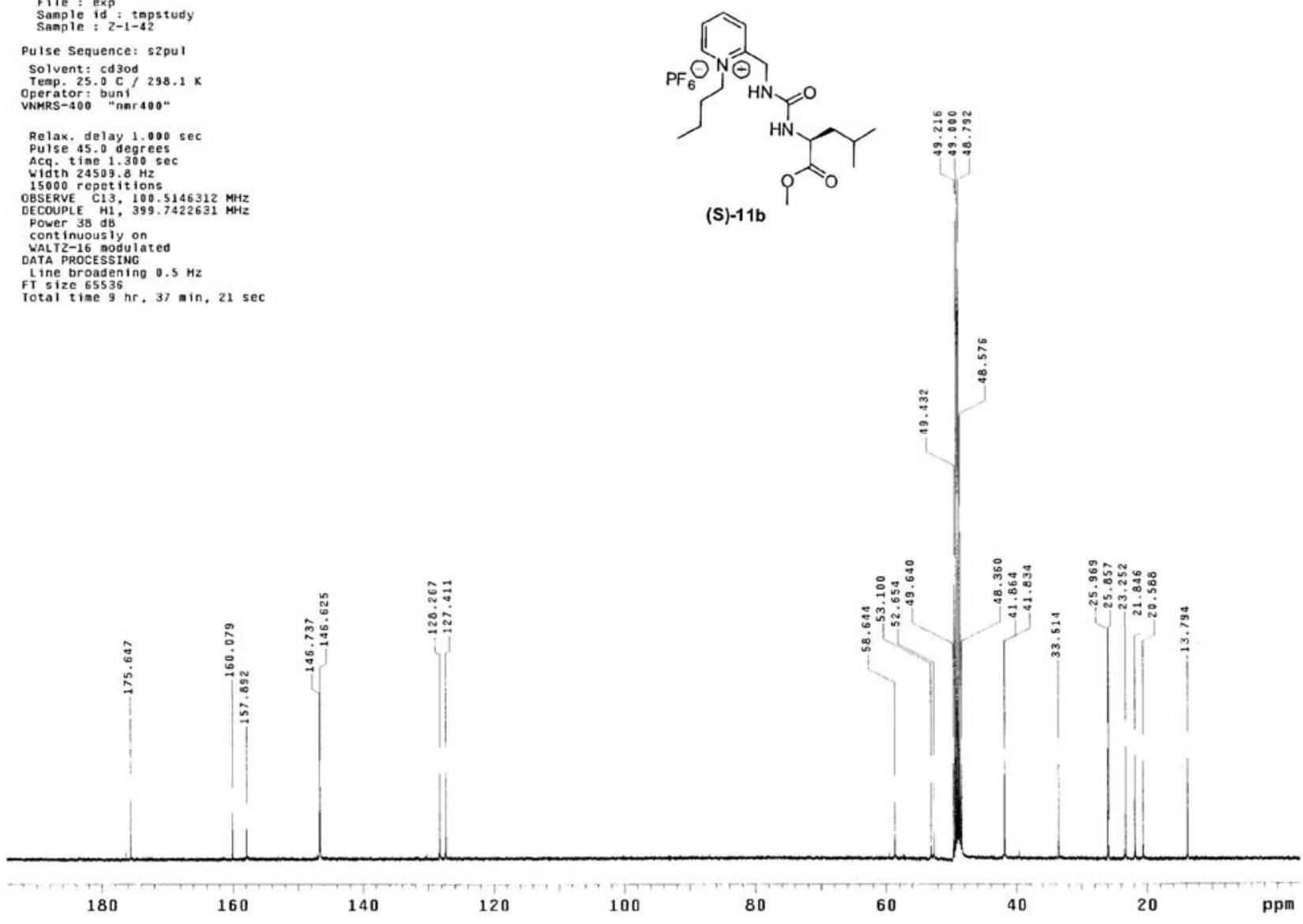




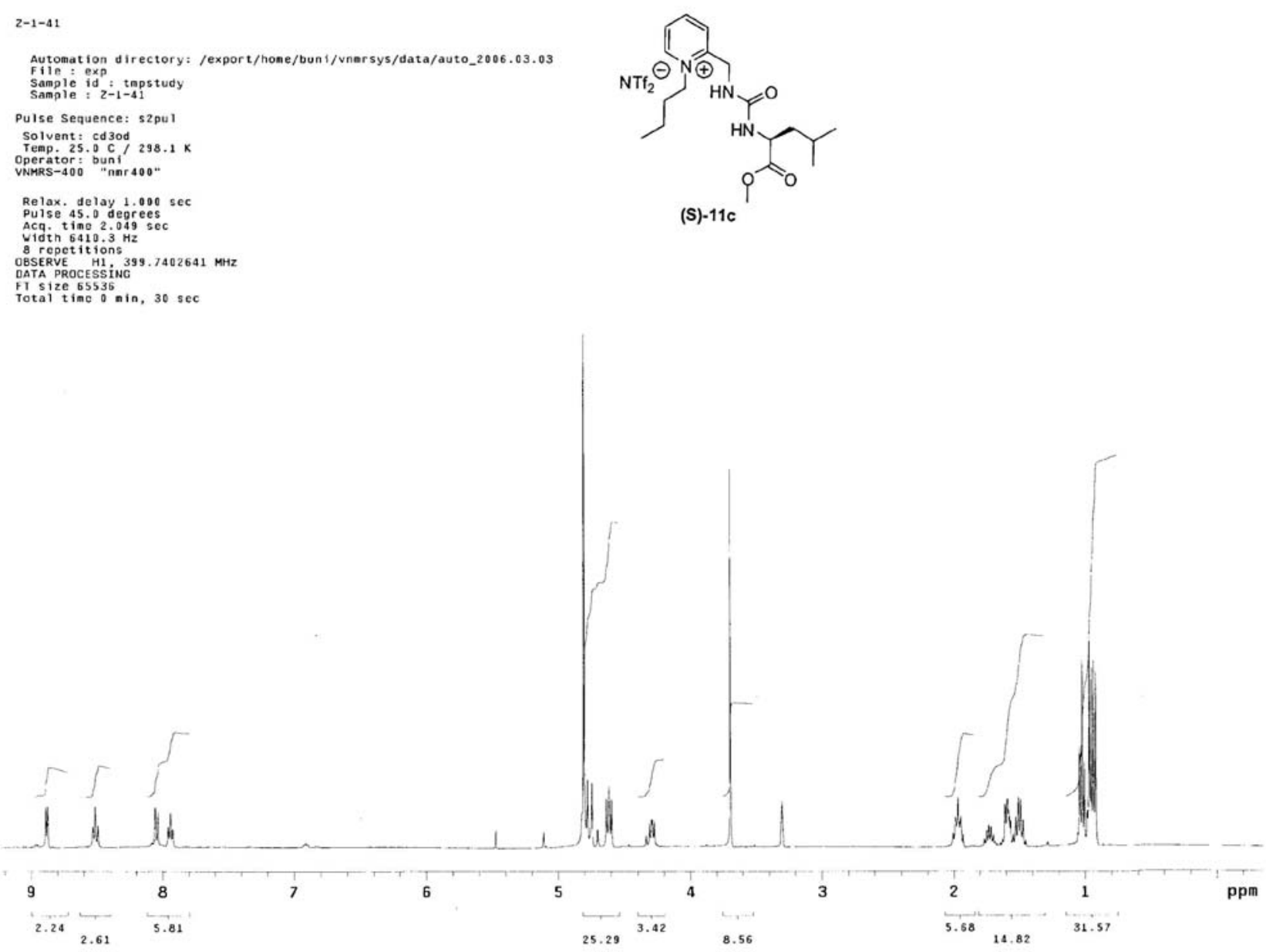


2-1-41

Automation directory: /export/home/buni/vnnrsys/data/auto_2005.03.03

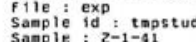

ise sequence: $52 \mathrm{pot}$

Solvent: cd3od $29.1 \mathrm{~K}$
Teap. $25.0 \mathrm{C} / 298.1 \mathrm{~K}$

OPRMRS -400 " Bun "nm 400

Relax. delay 1.000 sec
Pulse 45.0 degrees

Acq
Vidith $24509.8 \mathrm{~Hz}$

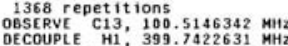

Power 38 od dB.
continuous ly on

Fif
Total tine 6 hr, $24 \mathrm{~min}, 54 \mathrm{sec}$

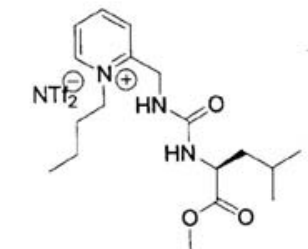

(S)-11c

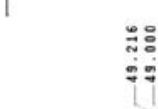

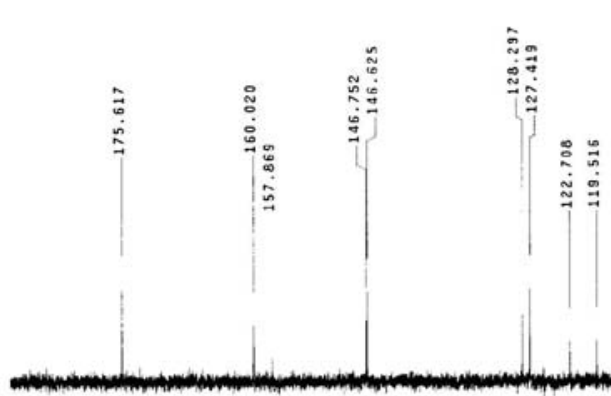

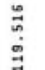

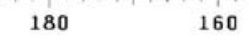

140

120

100

20

ppm 


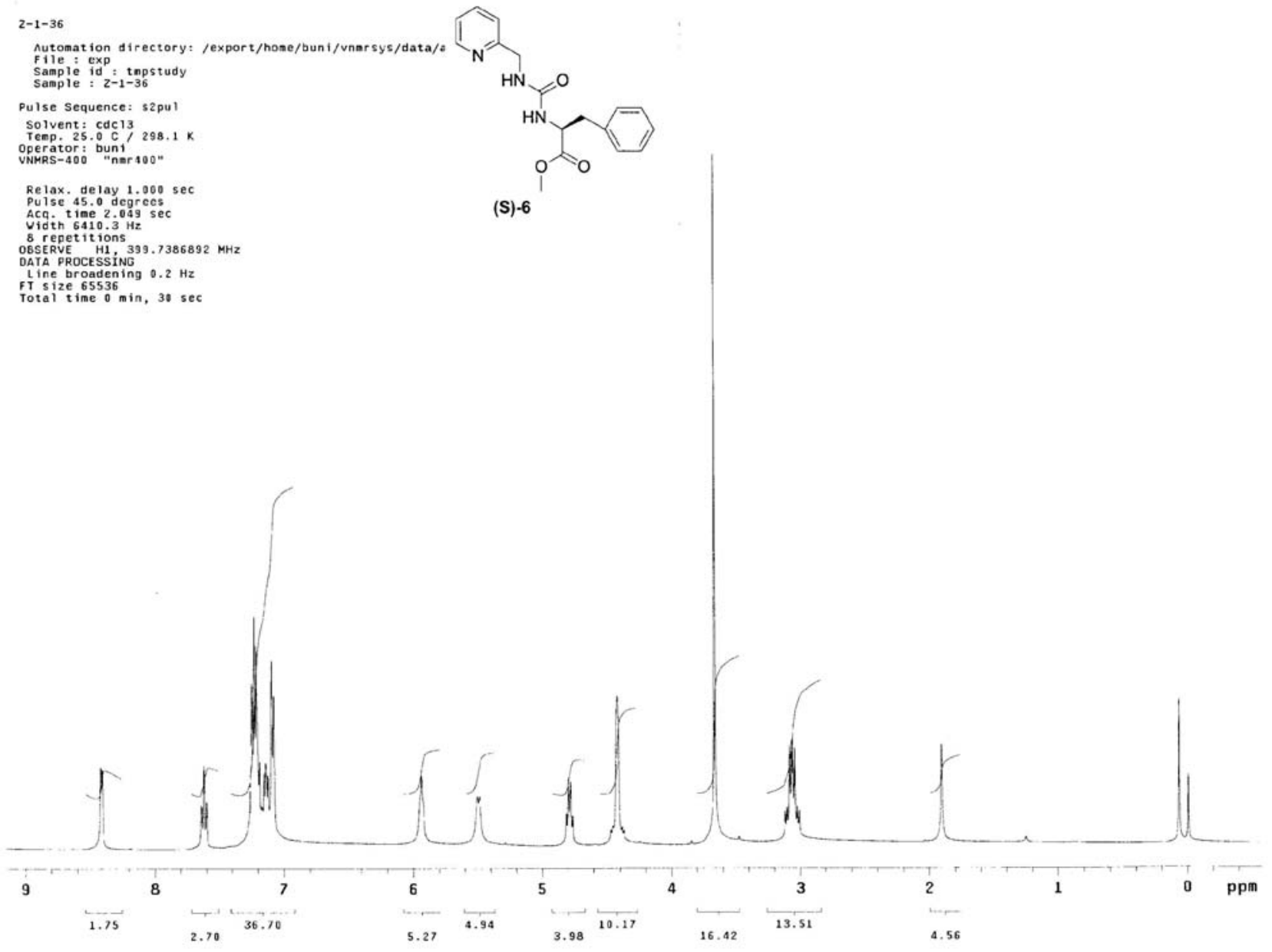

S26 
2-1-36

Automat ion directory: /export/home/buni/ynar sys/data/auto_2006.03.03 File : exp: tapstudy
sample id id : to
sample : $2-1-36$

Pulse Sequence: 52 pu 1

Solvent: cdclis $298.1 \times$

Operator: bunt
WNRRS -400 "nmar $400-$

Relax. delay 1.000 sec
Pulse 45.0 deorees
Acs time

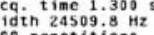

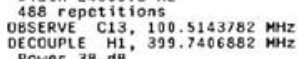

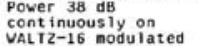

DATA PROCESSING

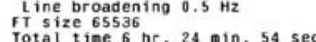

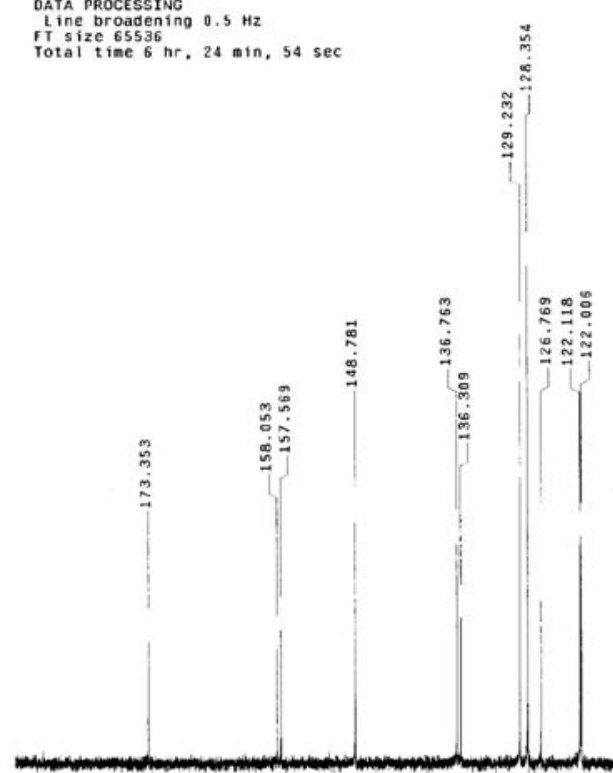

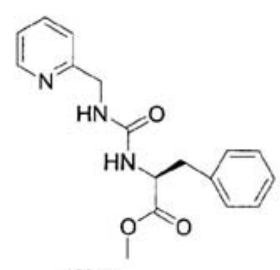

(S)-6

స్లి: ถ๊

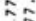

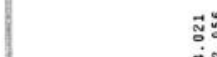

సี.

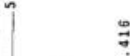

180

160 
2-1-96

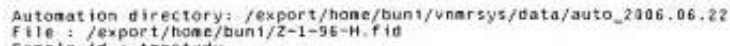

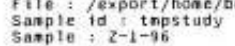

Pulse sequence: 82 pu

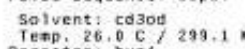

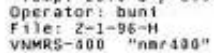

Relax. delay t, 000 see
Duise 45.0 degrees

$A c 9$ tine $2.0495 \mathrm{sec}$
vidin $6410.9 \mathrm{~Hz}$

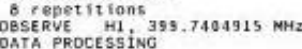

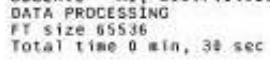

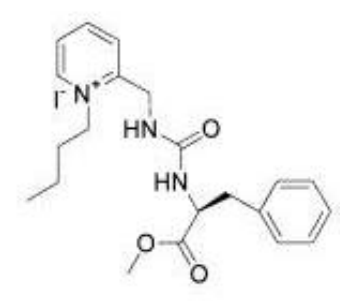

(S)- 9

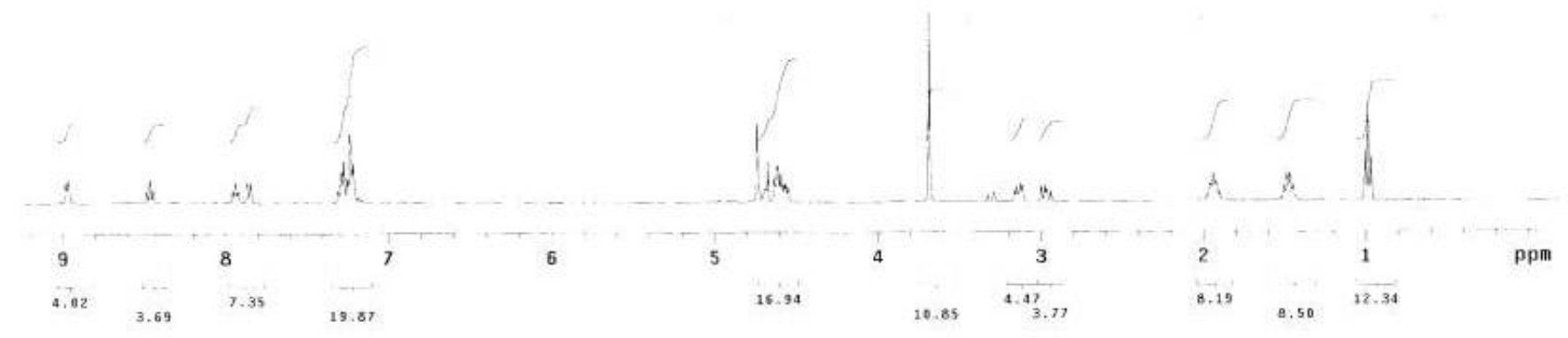


Autoaation directory: /export/hoae/buni/vnarsys/data/auto_2006.06.22

Saaple id $z$ tapstudy

Pulse Sequence: szoul

Solvent: cdsod

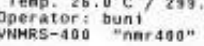

Relax. delay 1.000 sec

vidien $24509.8 \mathrm{~Hz}$

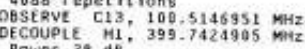

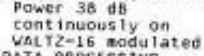

tine broadening $0.5 \mathrm{~Hz}$

rotal tian $12 \mathrm{hr}, 49 \mathrm{~min}, 40 \mathrm{sec}$

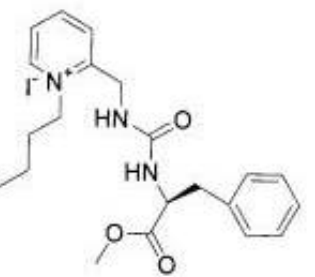

(S)- 9

:

क

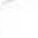

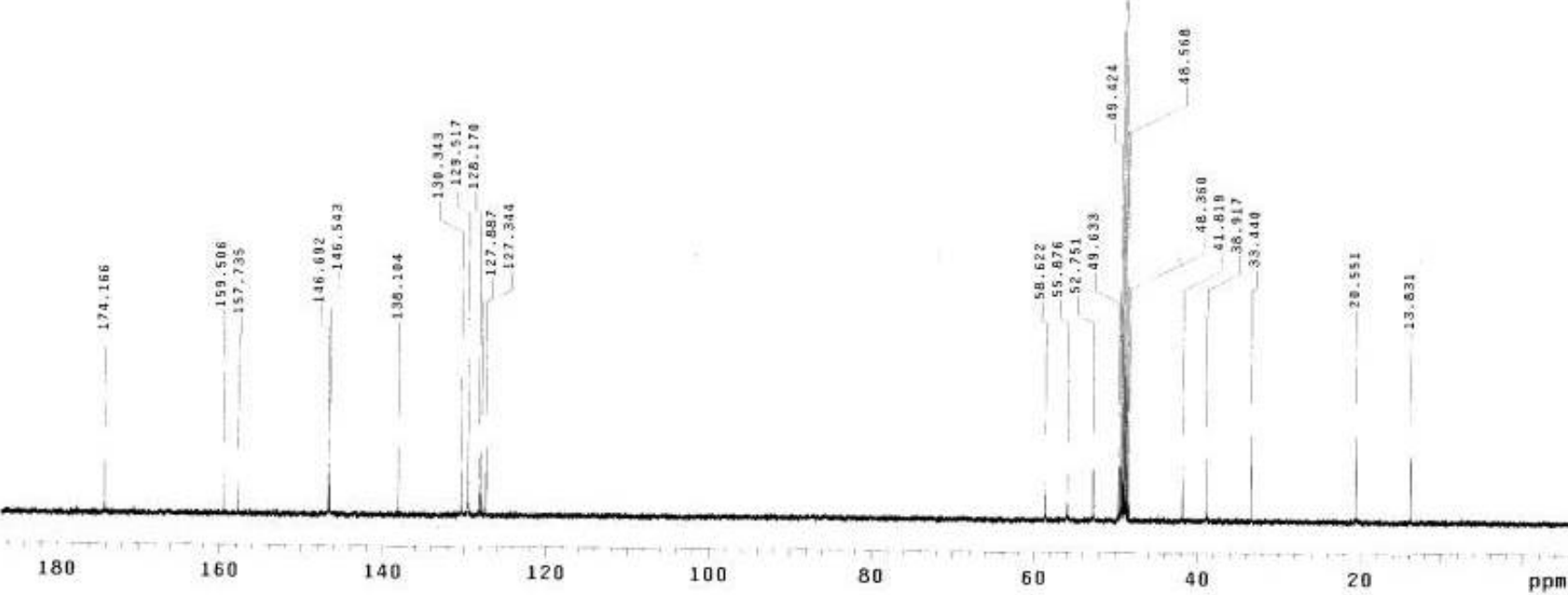


$2-1-46$

Automation directory: /export/home/buni/vnarsys/data/auto_2006. 03.03 mple id : tapstudy equence: $52 p u 1$ Temp. $25.0 \mathrm{C}$ C/298.

lax. delay 1.000

Pulse d5. doegrees
Act. tine 2.049

Single 5 scan
SSERVE

Ine broadenting $0.2 \mathrm{~Hz}$

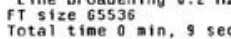

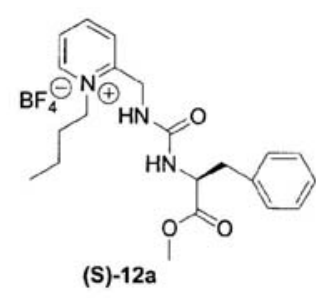

(S)-12a

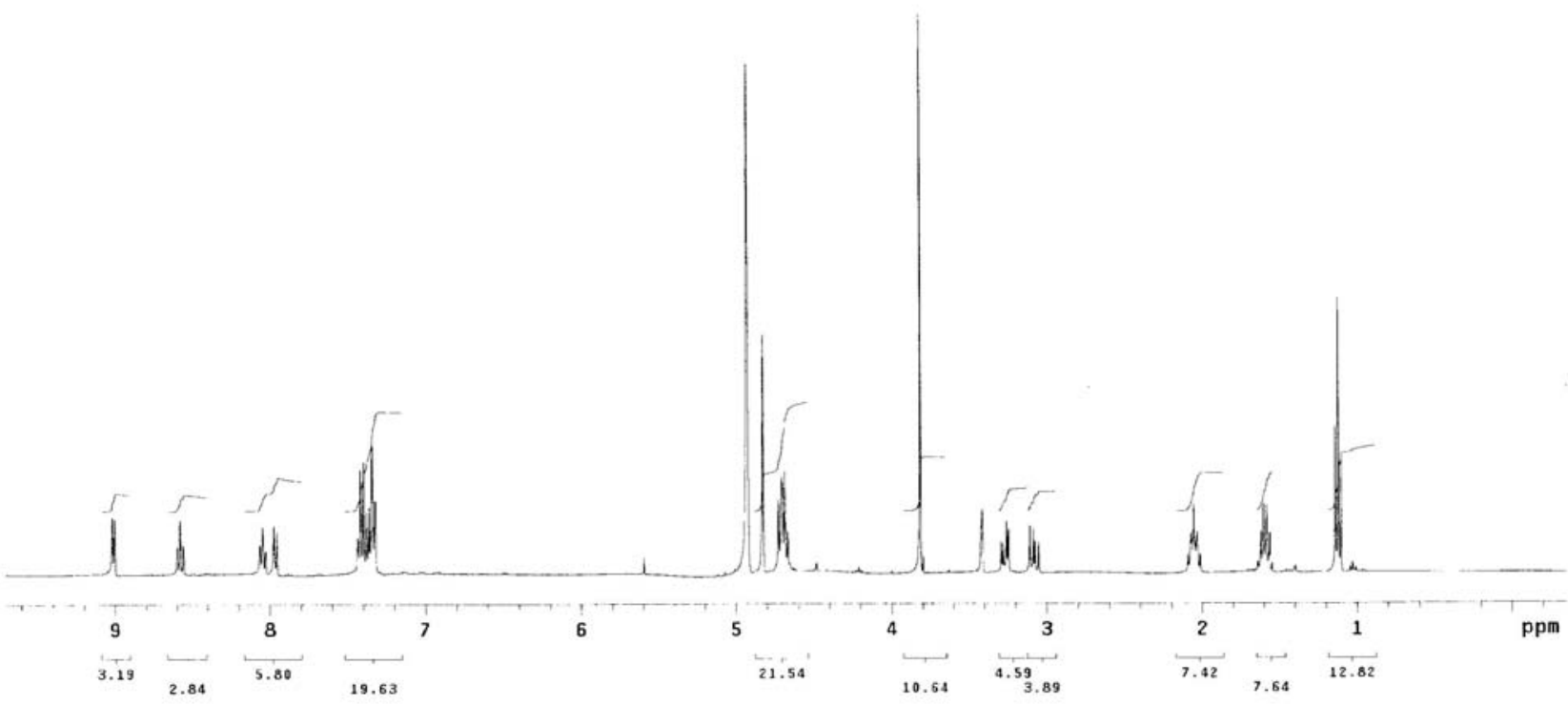


2-1-46

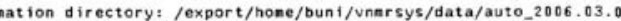

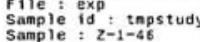
Pulse Sequence: s2pu

Solvent: ed 30 sod 298.1

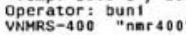

Relax. delay 1,000 sec
Pulse 45.0 degrees

iditin $2450.3 \mathrm{~Hz}$

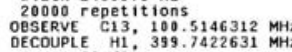

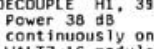

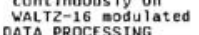

FI Size 55535 .

Total time $12 \mathrm{hr}, 49 \mathrm{gin}, 48$ sec

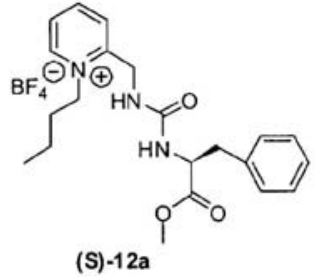

(S)-12a
影

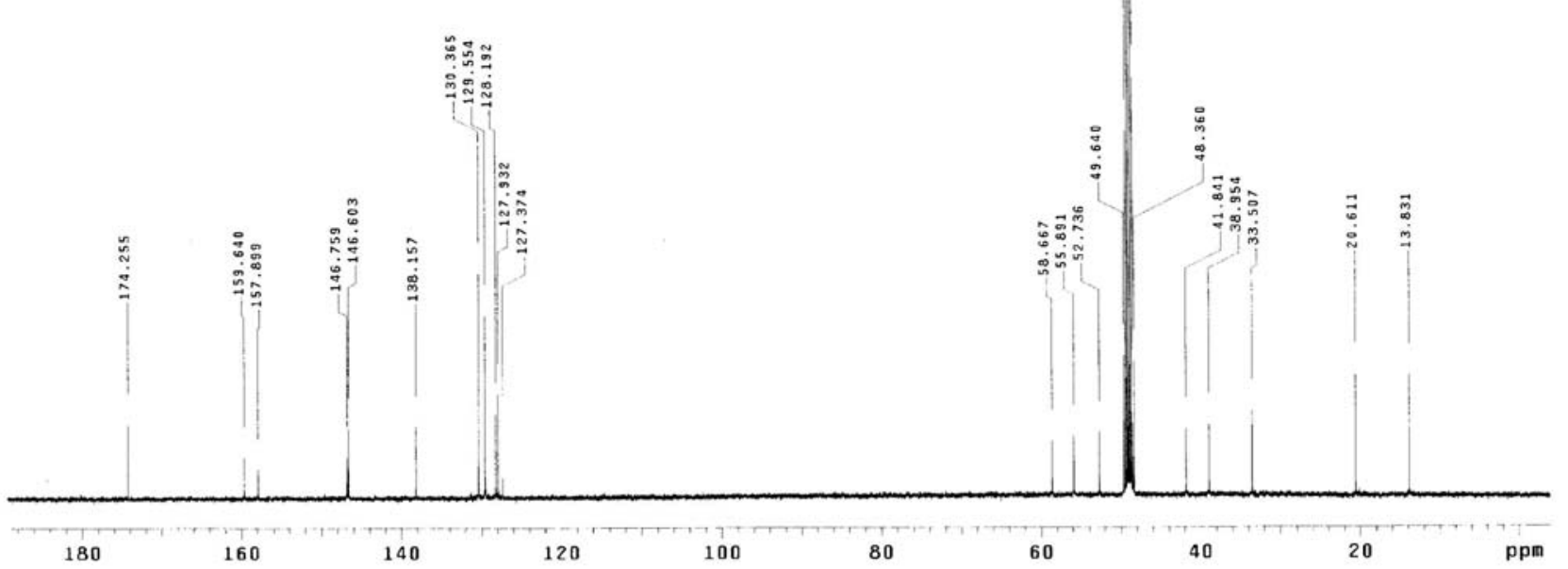




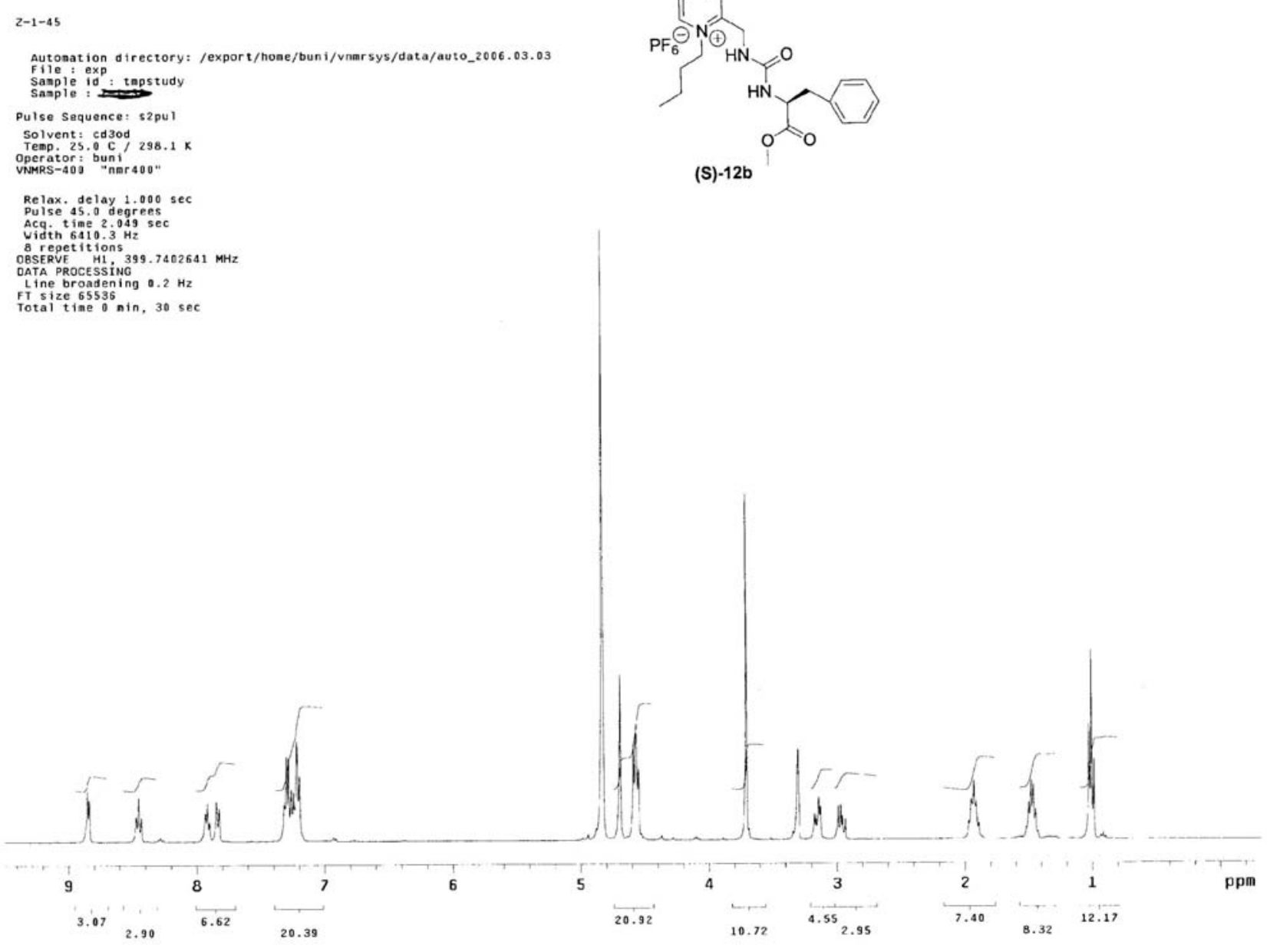

S32 
$20+1=42-1-43$

Automation directory: /export/hone/buni/vnmrsys/data/auto_2006.03.03

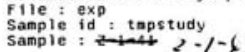
Pulse Sequence: s2pul

Solvent: cd cod
Temp. $25.0 \mathrm{C} / 298.1 \mathrm{~K}$

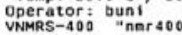

Relax. delay $1.000 \mathrm{sec}$
Pulse 45.0 degrees

Acg.

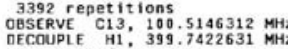

Power 38 di
cont inuously on

Fr size $65536 \mathrm{~s}$
Total tine $6 \mathrm{hr}, 24 \mathrm{~min}, 54 \mathrm{sec}$
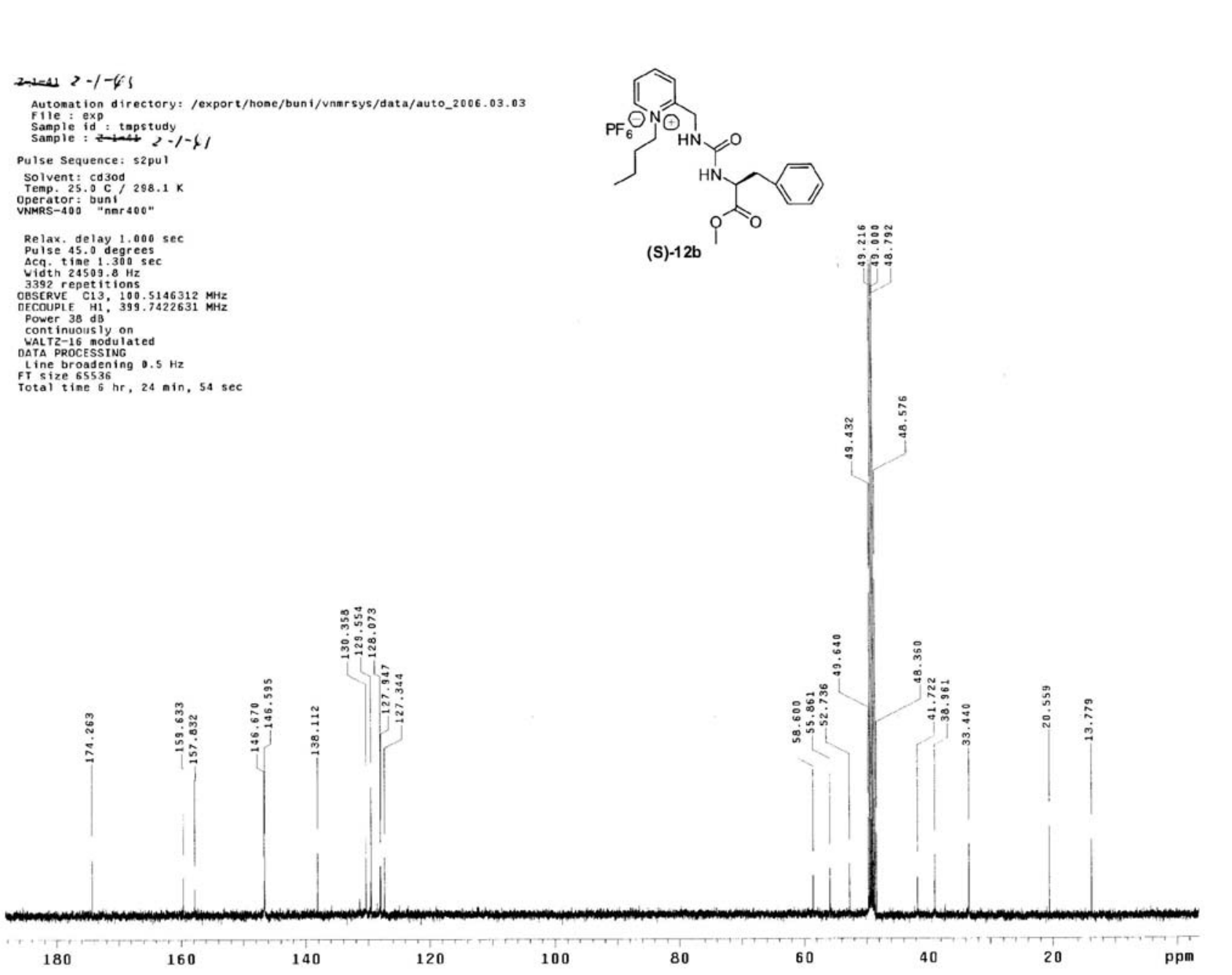


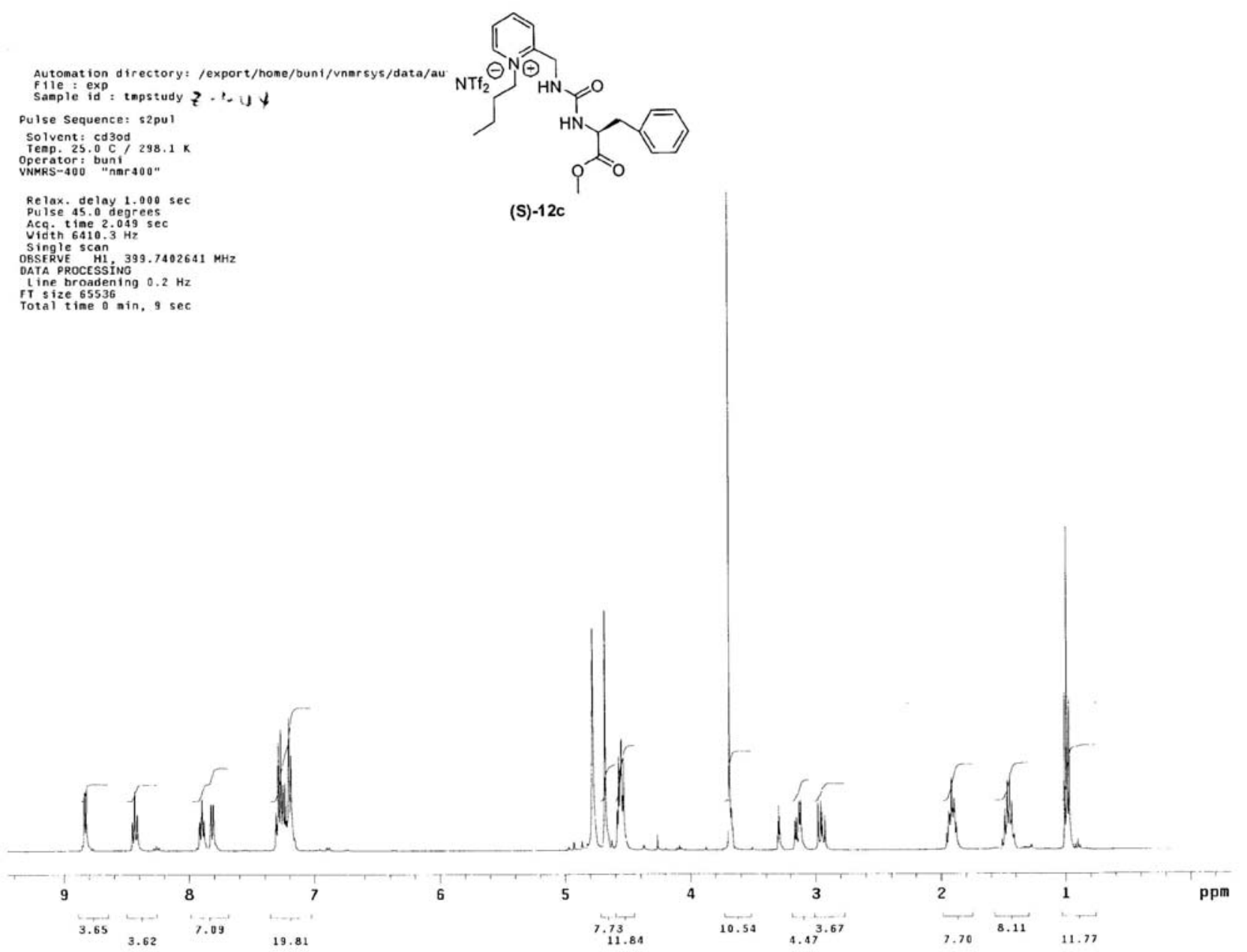


2-1-44

Automation directory: /export/home/buni/vnarsys/data/auto_2006.03.03

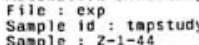

Pulse Sequence: s2pul

Solvent: cd cd 300
Temp. $25.0 \mathrm{C} / 298.1 \mathrm{~K}$

Operator:
VNMRS -400

Relax. delay $1.000 \mathrm{sec}$
Pulse 45.0 degrees

Acg time 1.300
Gidto $24509.8 \mathrm{~Hz}$

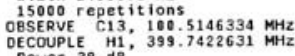

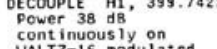

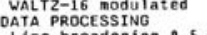

FT S size
Total time $956 \mathrm{hr}, 37 \mathrm{~min}, 21 \mathrm{sec}$

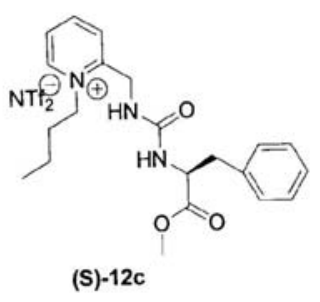

$\stackrel{\circ}{\longrightarrow}$

$: \mathfrak{x}^{\circ}$

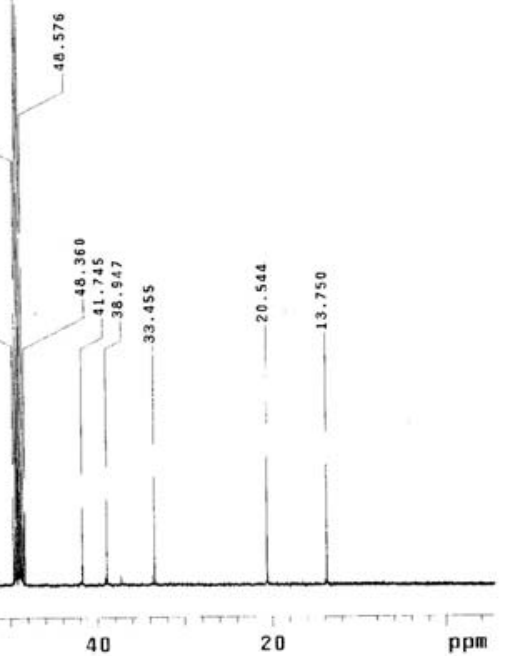

